\title{
Combining Ability, Heritability and Heterosis Estimates in Faba Bean (Vicia faba L.) under Two Water Regimes
}

\author{
Soad A. Mahmoud ${ }^{(1)}$, Amal M. Abd EL-Mageed ${ }^{(2)}$ and Enas S. Ibrahim ${ }^{(2) \#}$ \\ (1) Agronomy Department, Faculty of Agriculture, Suez Canal University, Ismailia, \\ Egypt; (2) Botany Department, Faculty of Agriculture, Suez Canal University Ismailia, \\ Egypt.
}

\begin{abstract}
$\mathrm{N}$ ORDER to study drought tolerance among faba bean genotypes, five cultivars of faba bean, Giza 843 (P1), Giza 3 (P2), Maser 3 (P3), Sakha 3 (P4) and Sakha 1 (P5) were used for carrying out half diallel mating design in 2014/2015 winter season to study combining ability, heritability and heterosis for growth, leaf anatomical, photosynthetic pigments and yield characteristics under stress condition. Parents and their crosses were evaluated in a yield trial, in 2015/2016 winter season under two water regimes which were well-watered $(100 \%$ from ETo) and severe water stress (60\% from ETo) at the experimental farm of the Faculty of Agriculture, Suez Canal University, Ismailia, Egypt. Results were showed highly significant of general (GCA) and specific (SCA) combining ability for all measured characters under two water deficit, indicating that GCA and SCA were important in the inheritance of these traits. The results of studied characters were indicated that the majority of crosses exhibited highly significant heterosis estimates for mid parents (MP). Heritability in narrow sense was low to moderate and ranged from 1.68 to $58.6 \%$. These results showed that these characters were greatly influenced by dominance and environmental condition. All preceding parents were studied to be good general combiners for its distinct characters. Also, results recommend that three of parental genotypes; P5, P2 and P4 were good combiners to improve the most characters and could be used as genetic resources for drought tolerance. The cross P1xP5 showed good SCA effects and significant values of heterosis for most characters under two water regimes. Also, the three crosses, $\mathrm{P} 3 \mathrm{xP} 4$ (for growth characters), $\mathrm{P} 1 \mathrm{xP} 2$ for (anatomical characters) and P2xP5 (for photosynthetic pigments characters and seed yield/plant) exhibited desirable SCA effects and significant heterosis values under two water regimes. These crosses might be used in faba bean breeding programs to produce pure lines have high yielding ability and drought tolerance.
\end{abstract}

Keywords : Water stress, Faba bean, Combining ability, Heterosis, Heritability, Leaf anatomy.

\section{Inroduction}

Each crop has different responses to environmental stresses such as drought. Major challenge for plant breeders is the improving of genetic resistance and different mechanisms for drought tolerance (Chaves et al., 2003). Faba bean is an important grain legume for protein (ranges from 20 to 40\%) security of demographically expanding and climatically changing world (Bishnoi et al., 2012). It has important value in improving the soil fertility by fixing nitrogen (Bishnoi et al., 2018a). Faba bean is a diploid $(2 n=2 x=12)$ autogamous annual plant with partial allogamy ranging from 20 to $80 \%$. The degree of out crossing depends on pollinator insect population and environmental conditions. Heterozygosity increase yield due to outcrossing has been well supported in faba bean. In view of, heterosis, resulting from the combined action and interaction of interallelic and allelic genes improved yield and obtained by hybrid combinations (Bishnoi et al., 2012 and Ibrahim, 2010).

Faba bean production isn't enough to feed the ever-growing world population. Numerous biotic and abiotic factors were caused reducing of yield (Abdelmula et al., 1999 and Abdelmula et al., 2012). Especially, Water deficit is the most important environmental and detrimental factor that decrease growth and productivity of plants

"Corresponding author email: enassafaa2010@hotmail.com 
with an average yields loss for most strategically crops by more than 50\% (Bray, 1997). Faba bean cultivation is unsuitable particularly in arid and semi-arid regions because this crop is not sufficiently water deficit tolerant (Loss et al., 1997). Also, bean plants exhibit a great quantity of Agro-physiological and biochemical modifications occur for drought tolerance (Jaleel et al., 2008; Farooq et al., 2008; Khan et al., 2010; Cortés et al., 2012; Ammar et al., 2014; Khazaei, 2014; Mohamed, 2015; Siddiqui et al., 2015; Abid et al., 2017; Ammar et al., 2017 and Ping et al., 2018).

In addition, inhibitive effect of water deficit supply on anatomical structure (lamina thickness and size of main vascular bundle) of tomato leaves. Moreover, the decrease in lamina thickness under drought stress was mostly due to the decrement induced in both palisade and spongy tissues (Selim \& El-Nady, 2011).

Combining ability had an important role in crop improvement. It could determine the magnitude and nature of genetic effects which controlling yield traits, in addition preparation the promising parents to use in the creation of genetic variability for eventual use in varieties improvement. Diallel analysis was an excellent means of obtaining information about differential parents and parental combinations in terms of general combining ability (GCA) and specific combining ability (SCA) (Griffing, 1956). The correspondence of better combining genotypes is conclusive for successful exploitation of heterosis. Also, evaluation of parental genotypes is essential for enveloping better hybrids. The estimates of combining ability effects supply important penetrative in selection of parents that could give rise to better hybrids upon crossing. Besides, the knowledge about the nature and magnitude of gene effects is importance for developing high yielding ability of varieties in faba bean (Beyene, 2016 and Ibrahim, 2010). Present investigation was carried out to understand; the type of gene action governing growth, anatomical, photosynthetic pigments and yield characters under water stress and detection good parents and hybrids, which could be exploited for future breeding programs.

\section{Materials and Methods}

This study was carried out during the two growing seasons of 2014/2015 and 2015/2016 at the experimental farm of the Faculty of Agriculture, Suez Canal University, Ismailia, Egypt. Five different local faba bean (Vicia. faba L.) cultivars, Giza 843 (P1), Giza 3 (P2), Maser 3 (P3), Sakha 3 (P4) and Sakha 1 (P5), were used in this study representing a wide range of variability in their agronomic traits. In the winter season of 2014/2015, the seeds of all parents were sown on $30^{\text {th }}$ October and crossed in a half diallel mating design to produce $10 \mathrm{~F} 1$ hybrids. In 2015/2016 season, the five parental genotypes and their crosses were sown under two water regimes.

\section{Meteorological data and irrigation treatments}

Relative humidity (RH), evaporation (E0), maximum and minimum temperature and solar radiation were recorded daily from sowing until harvest by a weather station located approximately $1 \mathrm{~km}$ from the experimental field. The temperature average about $16.8^{\circ} \mathrm{C}$ and the relative humidity average about $56 \%$ during winter 2015/2016 season were used for calculating reference crop evapotranspiration (ETo) according to Penman-Monteith equation (Allen et al., 1998). Irrigation water was supplied by surface to provide the two water regimes which were well-watered $(100 \%$ from ETo $)$ and severe water stress ( $60 \%$ from ETo), which represented 2800 and $1680 \mathrm{~m}^{3}$ of water/faddan, respectively. Each experimental plot consisted of three ridges of $3 \mathrm{~m}$ length and $60 \mathrm{~cm}$ width. Hills were spaced $20 \mathrm{~cm}$ with two plants per hill. All other agricultural practices were followed as recommended. A split-plot arrangement in randomized complete block design (RCBD) with three replications was used. Main plots were devoted to water treatments. Sub-plots were devoted to 15 genotypes (five parents and ten crosses).

\section{Measurements}

Data were recorded on ten competitive plants (five guarded hills) from each plot in each replications for the following four main group characters:

\section{1- Growth characters}

Number of leaves/plant, number of branches/ plant, shoot fresh weight/plant $(\mathrm{g})$, shoot dry weight/plant (g), root fresh weight/plant (g) and root dry weight/plant $(\mathrm{g})$. 


\section{2- Anatomical characters}

Number of xylem vessels, thickness of mysophyll $(\mu \mathrm{m})$, thickness of midrib $(\mu \mathrm{m})$, thickness of palisade tisuue $(\mu \mathrm{m})$, thickness of spongy tisuue $(\mu \mathrm{m})$ and thickness of vascular bundle $(\mu \mathrm{m})$ were determined.

The third visible leaf from the plant apex of five parental genotypes and their crosses at flowering stage was used to study the anatomy of the leaves. Killing and fixation of leaf sample in $70 \%$ F.A.A. solution, dehydration and clearing with ethyl-alcohol and xylene, infiltration and embedding in pure parafine wax (M. P. $56-58^{\circ} \mathrm{C}$ ) were carried out as described by Nassar \& ElSahhar (1998). Using a rotary microtome, sections of leaf $(15 \mu)$ were obtained and stained with safranin and light green. Sections, in such cases were microscopically examined and analyzed with the image processing program. Anatomical examination and measurements were achieved using a Leica light Research Microscope model PN: DM 500/13613210 supplied with a digital camera.

\section{3- Photosynthetic pigments}

Chlorophyll a, b and carotenoids were determined according to Fadeel (1962).

\section{4- Seed yield per plant (g)}

Some measurements about seed yield per plant (g) were determined

\section{Statistical analysis}

Testing the significance of genotypic differences: Data collected was initially subjected to analyses of variance (ANOVA) according to Steel \& Torrie (1980) using the COSTAT system for Window, version 6.311 (cohort software, Berkeley, CA, USA). Griffing (1956) model; method II, analysis was used to estimate general combining ability (GCA) and specific combining ability (SCA). The analyses were performed using the Diallel 98 program software computer package (Ukai, 2002). Heritability in narrow sense h (n.s) was calculated according to Mather \& Jinks (1982).

\section{Heterosis:}

Heterosis was estimated according to mid parent.

$\%$ Heterosis $(\mathrm{MP})=\frac{F_{1}-m \cdot P}{m \cdot \bar{p}} \times 100$

A test of significance for the F1 crosses mean from the mid parent values was calculated according to Bhatt (1971).

\section{Results and Discussion}

Mean performance of genotypes

The mean performances for studied characters of faba bean genotypes under two water regimes are given in Tables 1, 2, 3 and 4. Mean comparison were performed by least significant differences (LSD)

\section{Growth characters}

The data in Table 1 revealed to significant decreasing effects on growth performance of studied genotypes by water deficit. This decreasing was resulted from several inhibited changes of cell metabolisms (Farooq et al., 2012). The parent Giza 843 (P1) exhibited the highest values for number of leaves/plant and shoot fresh weight/plant (g) (70 and 115.7, respectively) under drought stress. The parent Giza 3 (P2) recorded high values for shoot dry weight/plant $(\mathrm{g})$ and root fresh weight/plant $(\mathrm{g})$ (19.7 and 19.7) under drought stress. For branches number/plant; the parental genotype Sakha1 (P5) recorded the highest value (3.3) under severe water stress. Concerning root dry weight/plant (g), the parental Maser 3 (P3) gave the highest value (3.8) under water stress.

With respect to the F1 crosses, results indicated that the cross $\mathrm{P} 1 \mathrm{xP} 2$ recorded high values forroot fresh weight/plant $(\mathrm{g})$ and root dry weight/plant $(\mathrm{g})$ (27.3 and 6.3, respectively) under severe drought stress. The cross $\mathrm{P} 1 \mathrm{xP} 4$ exhibited the highest values for shoot fresh weight/plant (g) and shoot dry weight/ plant (g) (203.3 and 35.7, respectively) under severe water stress. For number of leaves/plant the cross P1xP5 recorded the highest value (68) under severe water stress. Concerning number of branches/plant, the cross $\mathrm{P} 2 \mathrm{xP} 4$ gave the highest value (4.7) under severe water stress. These results suggest that the above mentioned parents and F1 crosses may be used for improving drought tolerance in faba bean breeding programs.

\section{Anatomical characters}

Data in Table 2 and Plates 1, 2 illustrated the values of number of xylem vessels, thickness of mesophyll $(\mu \mathrm{m})$, thickness of midrib $(\mu \mathrm{m})$, thickness of palisade tissue $(\mu \mathrm{m})$, thickness of spongy tissue $(\mu \mathrm{m})$ and thickness of vascular bundle $(\mu \mathrm{m})$. Faba bean parent P5 (Sakha 1) gave the maximum values in most of anatomical characters under two water regimes, followed by P2 (Giza 3). 
TABLE 1. Mean performance of faba bean genotypes for growth characters under water deficit.

\begin{tabular}{|c|c|c|c|c|c|c|c|c|c|}
\hline \multirow{2}{*}{ Genotypes } & \multicolumn{3}{|c|}{ No. of leaves/plant } & \multicolumn{3}{|c|}{ No. of branches/plant } & \multicolumn{3}{|c|}{ Shoot fresh weight/plant (g) } \\
\hline & $\begin{array}{c}100 \% \\
\text { ETo }\end{array}$ & $\begin{array}{l}60 \% \\
\text { ETo }\end{array}$ & Mean & $\begin{array}{c}100 \% \\
\text { ETo }\end{array}$ & $\begin{array}{l}60 \% \\
\text { ETo }\end{array}$ & Mean & $\begin{array}{c}100 \% \\
\text { ETo }\end{array}$ & $\begin{array}{l}60 \% \\
\text { ETo }\end{array}$ & Mean \\
\hline $\mathrm{P}_{1}$ & 84.3 & 70.0 & 77.2 & 5.0 & 2.0 & 3.5 & 260.0 & 115.7 & 187.9 \\
\hline $\mathrm{P}_{2}$ & 94.3 & 33.7 & 64.0 & 5.7 & 2.7 & 4.2 & 310.0 & 88.3 & 199.2 \\
\hline $\mathrm{P}_{3}$ & 70.3 & 50.3 & 60.3 & 7.0 & 2.7 & 4.9 & 258.3 & 98.3 & 178.3 \\
\hline $\mathrm{P}_{4}$ & 66.7 & 53.3 & 60.0 & 5.0 & 2.7 & 3.9 & 110.0 & 92.7 & 101.4 \\
\hline $\mathrm{P}_{5}$ & 51.0 & 38.7 & 44.9 & 2.0 & 3.3 & 2.7 & 100.0 & 95.0 & 97.5 \\
\hline $\mathrm{P}_{1} \mathrm{xP}_{2}$ & 91.7 & 66.7 & 79.2 & 5.0 & 3.0 & 4.0 & 260.0 & 188.3 & 224.2 \\
\hline $\mathrm{P}_{1} \mathrm{xP}_{3}$ & 80.7 & 62.3 & 71.5 & 4.3 & 3.7 & 4.0 & 188.3 & 130.0 & 159.2 \\
\hline $\mathrm{P}_{1} \mathrm{xP}_{4}$ & 116.0 & 37.3 & 76.7 & 6.3 & 3.7 & 5.0 & 216.7 & 203.3 & 210.0 \\
\hline $\mathrm{P}_{1} \mathrm{xP}_{5}$ & 95.7 & 68.0 & 81.9 & 6.0 & 3.3 & 4.7 & 355.0 & 165.0 & 260.0 \\
\hline $\mathrm{P}_{2} \mathrm{xP}_{3}$ & 81.7 & 43.3 & 62.5 & 4.0 & 2.7 & 3.4 & 250.0 & 195.0 & 222.5 \\
\hline $\mathrm{P}_{2} \mathrm{xP}_{4}$ & 93.3 & 45.0 & 69.2 & 6.0 & 4.7 & 5.4 & 210.0 & 155.0 & 182.5 \\
\hline $\mathrm{P}_{2} \mathrm{xP}_{5}$ & 86.7 & 63.3 & 75.0 & 5.0 & 3.0 & 4.0 & 140.0 & 100.0 & 120.0 \\
\hline $\mathrm{P}_{3} \mathrm{xP}_{4}$ & 109.7 & 55.0 & 82.4 & 7.3 & 4.0 & 5.7 & 393.3 & 140.0 & 266.7 \\
\hline $\mathrm{P}_{3} \mathrm{xP}_{5}$ & 145.0 & 45.0 & 95.0 & 9.0 & 3.0 & 6.0 & 450.0 & 64.7 & 257.4 \\
\hline $\mathrm{P}_{4} \mathrm{xP}_{5}$ & 112.7 & 54.3 & 83.5 & 5.7 & 2.3 & 4.0 & 330.0 & 61.7 & 195.9 \\
\hline Mean & 92.0 & 52.4 & 72.2 & 5.6 & 3.1 & 4.3 & 255.4 & 126.2 & 190.8 \\
\hline \multirow{2}{*}{$\operatorname{LSD}_{0.05}$} & Genot. & Water & $\mathrm{G} * \mathrm{~W}$ & Genot. & Water & $\mathrm{G} * \mathrm{~W}$ & Genot. & Water & $\mathrm{G} * \mathrm{~W}$ \\
\hline & 2.35 & 3.8 & 3.3 & 0.57 & 0.63 & 0.8 & 11.8 & 11.2 & 16.7 \\
\hline Characters & \multicolumn{3}{|c|}{ Shoot dry weight (g) } & \multicolumn{3}{|c|}{ Root fresh weight (g) } & \multicolumn{3}{|c|}{ Root dry weight (g) } \\
\hline Genotypes & $\begin{array}{c}100 \% \\
\text { ETo }\end{array}$ & $\begin{array}{l}60 \% \\
\text { ETo }\end{array}$ & Mean & $\begin{array}{c}100 \% \\
\text { ETo }\end{array}$ & $\begin{array}{l}60 \% \\
\text { ETo }\end{array}$ & Mean & $\begin{array}{c}100 \% \\
\text { ETo }\end{array}$ & $\begin{array}{l}60 \% \\
\text { ETo }\end{array}$ & Mean \\
\hline $\mathrm{P}_{1}$ & 43.7 & 18.7 & 31.2 & 45.7 & 14.3 & 30.0 & 14.3 & 3.3 & 8.8 \\
\hline $\mathrm{P}_{2}$ & 43.0 & 19.7 & 31.4 & 56.0 & 19.7 & 37.9 & 16.7 & 3.3 & 10.0 \\
\hline $\mathrm{P}_{3}$ & 39.7 & 19.0 & 29.4 & 27.3 & 17.3 & 22.3 & 8.0 & 3.8 & 5.9 \\
\hline $\mathrm{P}_{4}$ & 23.3 & 14.7 & 19.0 & 20.7 & 11.7 & 16.2 & 6.3 & 3.0 & 4.7 \\
\hline $\mathrm{P}_{5}$ & 19.7 & 13.0 & 16.4 & 18.0 & 9.0 & 13.5 & 5.0 & 2.5 & 3.8 \\
\hline $\mathrm{P}_{1} \mathrm{xP}_{2}$ & 33.7 & 27.7 & 30.7 & 37.7 & 27.3 & 32.5 & 11.7 & 6.3 & 9.0 \\
\hline $\mathrm{P}_{1} \mathrm{xP}_{3}$ & 37.3 & 30.3 & 33.8 & 35.0 & 21.7 & 28.4 & 13.0 & 4.3 & 8.7 \\
\hline $\mathrm{P}_{1} \mathrm{xP}_{4}$ & 42.7 & 35.7 & 39.2 & 34.3 & 19.0 & 26.7 & 15.3 & 3.0 & 9.2 \\
\hline $\mathrm{P}_{1} \mathrm{xP}_{5}$ & 62.3 & 34.3 & 48.3 & 27.7 & 16.7 & 22.2 & 11.0 & 3.5 & 7.3 \\
\hline $\mathrm{P}_{2} \mathrm{xP}_{3}$ & 37.3 & 28.3 & 32.8 & 27.7 & 20.7 & 24.2 & 9.0 & 4.3 & 6.7 \\
\hline $\mathrm{P}_{2} \mathrm{xP}_{4}$ & 37.0 & 27.7 & 32.4 & 33.3 & 19.0 & 26.2 & 11.0 & 5.2 & 8.1 \\
\hline $\mathrm{P}_{2} \mathrm{xP}_{5}$ & 52.7 & 32.3 & 42.5 & 28.3 & 23.0 & 25.7 & 8.7 & 4.0 & 6.4 \\
\hline $\mathrm{P}_{3} \mathrm{xP}_{4}$ & 65.0 & 26.3 & 45.7 & 42.0 & 17.0 & 29.5 & 10.0 & 3.8 & 6.9 \\
\hline $\mathrm{P}_{3} \mathrm{xP}_{5}$ & 62.7 & 31.7 & 47.2 & 65.0 & 17.7 & 41.4 & 24.3 & 4.0 & 14.2 \\
\hline $\mathrm{P}_{4} \mathrm{xP}_{5}$ & 56.3 & 22.0 & 39.2 & 30.0 & 13.0 & 21.5 & 13.3 & 2.8 & 8.1 \\
\hline Mean & 43.8 & 25.4 & 34.6 & 35.2 & 17.8 & 26.5 & 11.8 & 3.8 & 7.8 \\
\hline \multirow{2}{*}{$\operatorname{LSD}_{0.05}$} & Genot. & Water & $\mathrm{G}^{*} \mathrm{~W}$ & Genot. & Water & $\mathrm{G}^{*} \mathrm{~W}$ & Genot. & Water & $\mathrm{G}^{*} \mathrm{~W}$ \\
\hline & 2.8 & 3.2 & 4.0 & 2.4 & 0.25 & 3.4 & 0.63 & 0.41 & 0.9 \\
\hline
\end{tabular}

Egypt. J. Agron. 40, No.3 (2018) 
TABLE 2. Mean performance of faba bean genotypes for anatomical characters under water deficit.

\begin{tabular}{|c|c|c|c|c|c|c|c|c|c|}
\hline \multirow{2}{*}{ Genotypes } & \multicolumn{3}{|c|}{ No. of xylem vessels } & \multicolumn{3}{|c|}{ Thickness of mesophyll $\mu \mathrm{m}$} & \multicolumn{3}{|c|}{ Thickness of midrib $\mu \mathrm{m}$} \\
\hline & $\begin{array}{l}100 \% \\
\text { ETo }\end{array}$ & $\begin{array}{l}60 \% \\
\text { ETo }\end{array}$ & Mean & $\begin{array}{l}100 \% \\
\text { ETo }\end{array}$ & $\begin{array}{l}60 \% \\
\text { ETo }\end{array}$ & Mean & $\begin{array}{c}100 \% \\
\text { ETo }\end{array}$ & $\begin{array}{l}60 \% \\
\text { ETo }\end{array}$ & Mean \\
\hline $\mathrm{P}_{1}$ & 11.0 & 13.7 & 12.4 & 299.0 & 271.0 & 285.0 & 607.3 & 581.0 & 594.2 \\
\hline $\mathrm{P}_{2}$ & 12.3 & 16.0 & 14.2 & 470.0 & 392.7 & 431.4 & 668.7 & 751.0 & 709.9 \\
\hline $\mathrm{P}_{3}$ & 12.3 & 14.3 & 12.3 & 413.7 & 428.7 & 421.2 & 709.7 & 628.7 & 669.2 \\
\hline $\mathrm{P}_{4}$ & 15.3 & 10.7 & 13.0 & 528.0 & 357.0 & 442.5 & 784.7 & 785.7 & 785.2 \\
\hline $\mathrm{P}_{5}$ & 21.0 & 15.7 & 18.4 & 607.0 & 521.0 & 564.0 & 941.7 & 900.3 & 921.0 \\
\hline $\mathrm{P}_{1} \mathrm{xP}_{2}$ & 14.7 & 19.0 & 16.9 & 928.3 & 500.0 & 714.2 & 535.3 & 850.7 & 693.0 \\
\hline $\mathrm{P}_{1} \mathrm{xP}_{3}$ & 11.0 & 17.3 & 14.2 & 406.0 & 428.7 & 417.4 & 577.3 & 786.0 & 681.7 \\
\hline $\mathrm{P}_{1} \mathrm{xP}_{4}$ & 11.0 & 16.3 & 13.7 & 413.0 & 485.7 & 449.4 & 585.7 & 878.7 & 732.2 \\
\hline $\mathrm{P}_{1} \mathrm{xP}_{5}$ & 14.0 & 16.0 & 15.0 & 334.0 & 414.0 & 374.0 & 750.0 & 906.7 & 828.4 \\
\hline $\mathrm{P}_{2} \mathrm{xP}_{3}$ & 11.7 & 17.0 & 14.4 & 614.0 & 506.7 & 567.5 & 771.0 & 715.0 & 743.0 \\
\hline $\mathrm{P}_{2} \mathrm{xP}_{4}$ & 11.3 & 15.0 & 13.2 & 777.7 & 435.7 & 606.7 & 928.7 & 750.3 & 839.5 \\
\hline $\mathrm{P}_{2} \mathrm{xP}_{5}$ & 10.0 & 13.0 & 11.5 & 500.3 & 435.0 & 467.7 & 678.0 & 763.3 & 720.7 \\
\hline $\mathrm{P}_{3} \mathrm{xP}_{4}$ & 15.0 & 15.0 & 15.0 & 443.0 & 357.0 & 400.0 & 642.3 & 628.7 & 635.5 \\
\hline $\mathrm{P}_{3} \mathrm{xP}_{5}$ & 20.0 & 15.0 & 17.5 & 350.7 & 428.7 & 389.7 & 743.0 & 642.3 & 692.7 \\
\hline $\mathrm{P}_{4} \mathrm{xP}_{5}$ & 15.0 & 10.0 & 12.5 & 585.3 & 392.7 & 489.0 & 891.0 & 714.0 & 802.5 \\
\hline Mean & 14.1 & 14.4 & 14.3 & 549.7 & 452.3 & 501.0 & 721.0 & 752.2 & 736.6 \\
\hline \multirow{2}{*}{$\operatorname{LSD}_{0.05}$} & Genot. & Water & $\mathrm{G} * \mathrm{~W}$ & Genot. & Water & $\mathrm{G} * \mathrm{~W}$ & Genot. & Water & $\mathrm{G} * \mathrm{~W}$ \\
\hline & 1.2 & 0.7 & 1.7 & 0.73 & 0.44 & 1.0 & 1.7 & 2.99 & 2.9 \\
\hline \multirow[t]{2}{*}{ Cha } & \multicolumn{3}{|c|}{$\begin{array}{c}\text { Thickness of palisade tisuue } \\
\mu \mathrm{m}\end{array}$} & \multicolumn{3}{|c|}{$\begin{array}{c}\text { Thickness of spongy tisuue } \\
\mu \mathrm{m}\end{array}$} & \multicolumn{3}{|c|}{$\begin{array}{c}\text { Thickness of vascular bundle } \\
\mu \mathrm{m}\end{array}$} \\
\hline & $\begin{array}{l}100 \% \\
\text { ETo }\end{array}$ & $\begin{array}{l}60 \% \\
\text { ETo }\end{array}$ & Mean & $\begin{array}{l}100 \% \\
\text { ETo }\end{array}$ & $\begin{array}{l}60 \% \\
\text { ETo }\end{array}$ & Mean & $\begin{array}{c}100 \% \\
\text { ETo }\end{array}$ & $\begin{array}{l}60 \% \\
\text { ETo }\end{array}$ & Mean \\
\hline $\mathrm{P}_{1}$ & 121.7 & 112.0 & 116.9 & 178.7 & 160.0 & 169.4 & 213.7 & 200.0 & 206.9 \\
\hline $\mathrm{P}_{2}$ & 142.7 & 114.7 & 128.7 & 328.7 & 278.7 & 303.7 & 235.7 & 214.0 & 224.9 \\
\hline $\mathrm{P}_{3}$ & 185.3 & 143.0 & 164.2 & 228.7 & 285.7 & 257.2 & 178.3 & 206.7 & 192.5 \\
\hline $\mathrm{P}_{4}$ & 164.3 & 121.7 & 143.0 & 363.7 & 235.7 & 299.7 & 285.7 & 185.7 & 235.7 \\
\hline $\mathrm{P}_{5}$ & 200.3 & 192.7 & 196.5 & 407.3 & 328.7 & 368.0 & 263.7 & 221.3 & 242.5 \\
\hline $\mathrm{P}_{1} \mathrm{xP}_{2}$ & 156.3 & 142.7 & 149.5 & 771.3 & 356.7 & 564.0 & 320.7 & 285.7 & 303.2 \\
\hline $\mathrm{P}_{1} \mathrm{xP}_{3}$ & 142.7 & 135.7 & 139.2 & 263.7 & 292.7 & 278.2 & 192.3 & 250.0 & 221.2 \\
\hline $\mathrm{P}_{1} \mathrm{xP}_{4}$ & 142.3 & 164.3 & 153.3 & 271.3 & 321.7 & 296.5 & 213.7 & 285.7 & 249.7 \\
\hline $\mathrm{P}_{1} \mathrm{xP}_{5}$ & 92.7 & 164.7 & 128.7 & 242.3 & 250.3 & 246.3 & 235.3 & 271.3 & 253.3 \\
\hline $\mathrm{P}_{2} \mathrm{xP}_{3}$ & 250.7 & 165.0 & 207.9 & 363.7 & 342.7 & 353.2 & 200.3 & 200.3 & 200.3 \\
\hline $\mathrm{P}_{2} \mathrm{xP}_{4}$ & 356.7 & 128.3 & 242.5 & 421.3 & 306.7 & 364.0 & 306.7 & 214.3 & 260.5 \\
\hline $\mathrm{P}_{2} \mathrm{xP}_{5}$ & 171.3 & 142.7 & 157.0 & 328.7 & 292.7 & 310.7 & 192.7 & 214.0 & 203.4 \\
\hline $\mathrm{P}_{3} \mathrm{xP}_{4}$ & 156.3 & 128.7 & 142.5 & 285.7 & 228.7 & 257.2 & 150.3 & 212.7 & 181.5 \\
\hline $\mathrm{P}_{3} \mathrm{xP}_{5}$ & 128.3 & 128.7 & 128.5 & 221.3 & 300.3 & 260.8 & 213.7 & 235.7 & 224.7 \\
\hline $\mathrm{P}_{4} \mathrm{xP}_{5}$ & 214.0 & 142.7 & 178.4 & 371.0 & 250.7 & 310.9 & 271.3 & 221.3 & 246.3 \\
\hline Mean & 175.0 & 141.8 & 158.4 & 336.5 & 282.1 & 309.3 & 231.6 & 227.9 & 229.8 \\
\hline \multirow{2}{*}{$\operatorname{LSD}_{0.05}$} & Genot. & Water & $\mathrm{G}^{*} \mathrm{~W}$ & Genot. & Water & $\mathrm{G}^{*} \mathrm{~W}$ & Genot. & Water & $\mathrm{G}^{*} \mathrm{~W}$ \\
\hline & 0.7 & 0.85 & 1.0 & 0.64 & 0.19 & 0.9 & 0.7 & 0.63 & 1.0 \\
\hline
\end{tabular}


TABLE 3. Mean performance of faba bean genotypes for photosynthetic pigments characters under water deficit.

\begin{tabular}{|c|c|c|c|c|c|c|c|c|c|}
\hline \multirow{2}{*}{ Characters } & \multicolumn{3}{|c|}{ Chlorophyll a } & \multicolumn{3}{|c|}{ Chlorophyll b } & \multicolumn{3}{|c|}{ Carotenoids } \\
\hline & $\begin{array}{c}100 \% \\
\text { ETo }\end{array}$ & $\begin{array}{l}60 \% \\
\text { ETo }\end{array}$ & Mean & $\begin{array}{c}100 \% \\
\text { ETo }\end{array}$ & $\begin{array}{l}60 \% \\
\text { ETo }\end{array}$ & Mean & $\begin{array}{c}100 \% \\
\text { ETo }\end{array}$ & $\begin{array}{l}60 \% \\
\text { ETo }\end{array}$ & Mean \\
\hline $\mathrm{P}_{1}$ & 2.1 & 2.6 & 2.4 & 3.6 & 4.5 & 4.1 & 1.5 & 2.3 & 1.9 \\
\hline $\mathrm{P}_{2}$ & 1.6 & 2.6 & 2.1 & 2.5 & 4.5 & 3.5 & 1.4 & 1.4 & 1.4 \\
\hline $\mathrm{P}_{3}$ & 4.2 & 4.4 & 4.3 & 3.3 & 7.8 & 5.6 & 1.3 & 1.5 & 1.4 \\
\hline $\mathrm{P}_{4}$ & 2.2 & 2.1 & 2.2 & 3.8 & 3.7 & 3.8 & 1.6 & 2.5 & 2.1 \\
\hline $\mathrm{P}_{5}$ & 3.3 & 3.1 & 3.2 & 6.2 & 5.4 & 5.8 & 1.7 & 1.5 & 1.6 \\
\hline $\mathrm{P}_{1} \mathrm{xP}_{2}$ & 1.4 & 2.2 & 1.8 & 2.4 & 3.7 & 3.1 & 1.6 & 1.8 & 1.7 \\
\hline $\mathrm{P}_{1} \mathrm{xP}_{3}$ & 1.8 & 3.4 & 2.6 & 3.1 & 5.8 & 4.5 & 2.2 & 1.4 & 1.8 \\
\hline $\mathrm{P}_{1} \mathrm{xP}_{4}$ & 2.5 & 1.3 & 1.9 & 4.3 & 2.2 & 3.3 & 0.8 & 1.8 & 1.3 \\
\hline $\mathrm{P}_{1} \mathrm{xP}_{5}$ & 3.6 & 3.1 & 3.4 & 6.3 & 5.3 & 5.8 & 0.2 & 1.5 & 0.9 \\
\hline $\mathrm{P}_{2} \mathrm{xP}_{3}$ & 3.2 & 1.8 & 2.5 & 4.4 & 3.2 & 3.8 & 0.4 & 1.4 & 0.9 \\
\hline $\mathrm{P}_{2} \mathrm{xP}_{4}$ & 3.8 & 1.8 & 2.8 & 6.7 & 3.4 & 5.1 & 1.3 & 2.8 & 2.1 \\
\hline $\mathrm{P}_{2} \mathrm{xP}_{5}$ & 3.4 & 1.8 & 2.6 & 4.5 & 3.6 & 4.1 & 1.8 & 2.3 & 2.1 \\
\hline $\mathrm{P}_{3} \mathrm{xP}_{4}$ & 1.8 & 1.9 & 1.9 & 2.8 & 3.4 & 3.1 & 0.8 & 1.4 & 1.1 \\
\hline $\mathrm{P}_{3} \mathrm{xP}_{5}$ & 2.4 & 3.2 & 2.8 & 4.1 & 5.6 & 4.9 & 1.6 & 1.7 & 1.7 \\
\hline $\mathrm{P}_{4} \mathrm{xP}_{5}$ & 3.2 & 2.2 & 2.7 & 5.6 & 3.5 & 4.6 & 1.5 & 1.9 & 1.7 \\
\hline Mean & 2.7 & 2.5 & 2.6 & 4.2 & 4.4 & 4.3 & 1.3 & 1.8 & 1.6 \\
\hline \multirow{2}{*}{$\operatorname{LSD}_{0.05}$} & Genot. & Water & $\mathrm{G}^{*} \mathrm{~W}$ & Genot. & Water & $\mathrm{G}^{*} \mathrm{~W}$ & Genot. & Water & $\mathrm{G}^{*} \mathrm{~W}$ \\
\hline & 0.08 & 0.03 & 0.1 & 0.06 & 0.04 & 0.1 & 0.08 & 0.04 & 0.1 \\
\hline
\end{tabular}

TABLE 4. Mean performance of faba bean genotypes forseed yield/plant (g) under water deficit.

\begin{tabular}{|c|c|c|c|}
\hline \multirow[b]{2}{*}{ Genotypes } & \multicolumn{3}{|c|}{ Seed yield/plant (g) } \\
\hline & $100 \%$ ETo & $60 \%$ ETo & Mean \\
\hline $\mathrm{P}_{1}$ & 100.0 & 50.3 & 75.2 \\
\hline $\mathrm{P}_{2}$ & 71.7 & 50.0 & 60.9 \\
\hline $\mathrm{P}_{3}$ & 110.0 & 34.0 & 72.0 \\
\hline $\mathrm{P}_{4}$ & 105.3 & 52.3 & 78.8 \\
\hline $\mathrm{P}_{5}$ & 40.0 & 31.7 & 35.9 \\
\hline $\mathrm{P}_{1} \mathrm{xP}_{2}$ & 63.3 & 42.0 & 52.7 \\
\hline $\mathrm{P}_{1} \mathrm{xP}_{3}$ & 82.7 & 51.3 & 67.0 \\
\hline $\mathrm{P}_{1} \mathrm{xP}_{4}$ & 130.0 & 70.0 & 100.0 \\
\hline $\mathrm{P}_{1} \mathrm{xP}_{5}$ & 180.0 & 76.7 & 128.4 \\
\hline $\mathrm{P}_{2} \mathrm{XP}_{3}$ & 100.0 & 27.0 & 63.5 \\
\hline $\mathrm{P}_{2} \mathrm{xP}_{4}$ & 125.0 & 26.3 & 75.7 \\
\hline $\mathrm{P}_{2} \mathrm{xP}_{5}$ & 122.3 & 52.7 & 87.5 \\
\hline $\mathrm{P}_{3} \mathrm{xP}_{4}$ & 100.0 & 42.7 & 71.4 \\
\hline $\mathrm{P}_{3} \mathrm{xP}_{5}$ & 135.0 & 51.0 & 93.0 \\
\hline $\mathrm{P}_{4} \mathrm{xP}_{5}$ & 93.3 & 49.0 & 71.2 \\
\hline Mean & 103.9 & 47.1 & 75.5 \\
\hline \multirow{2}{*}{ LSD $_{0.05}$} & Genot. & Water & $\mathrm{G}^{*} \mathrm{~W}$ \\
\hline & 3.4 & 2.3 & 4.9 \\
\hline
\end{tabular}



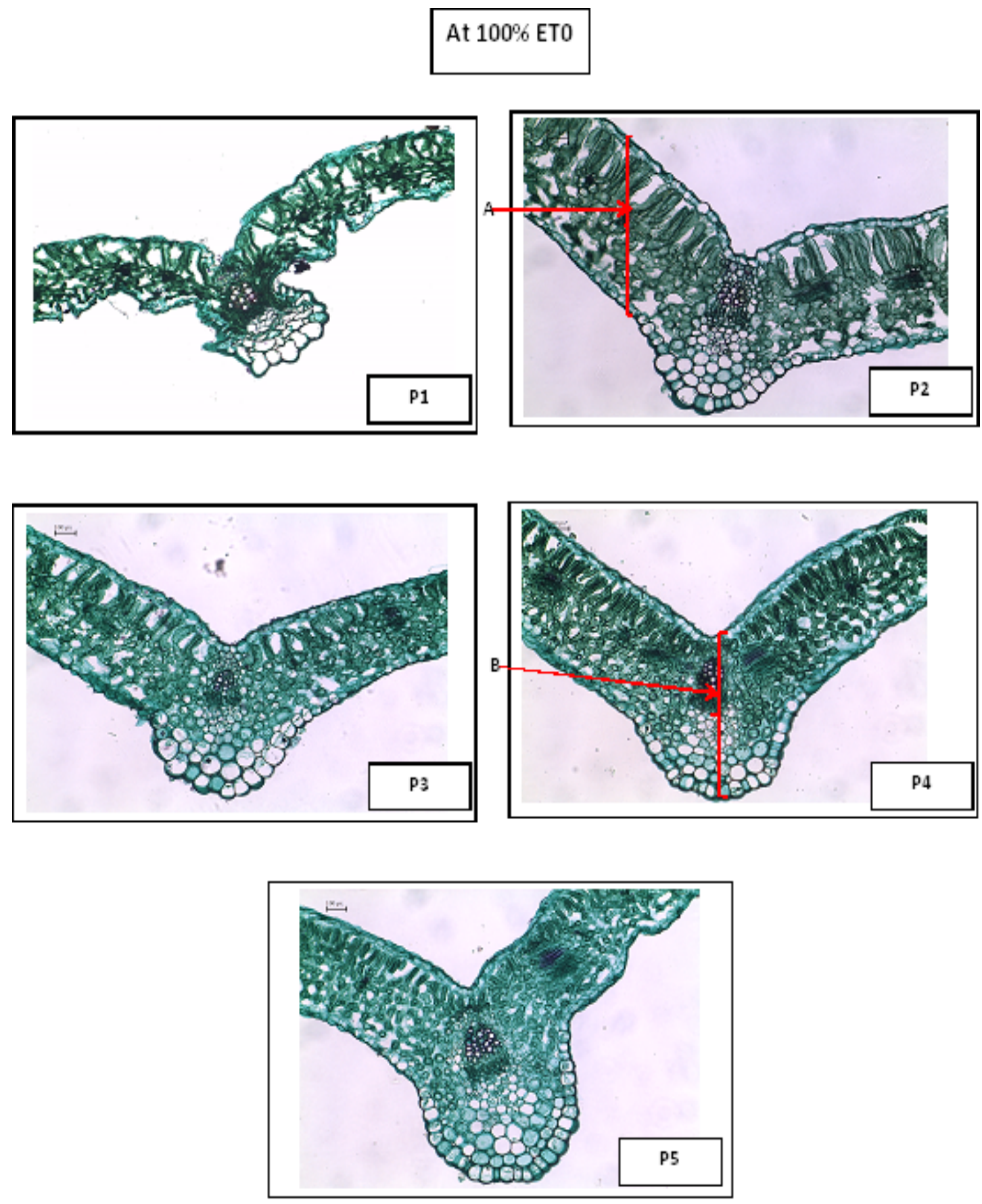

Plate 1. Cross sections of leaves of faba bean cultivars, Giza $843\left(P_{1}\right)$, Giza $3\left(P_{2}\right)$, Maser $3\left(P_{3}\right)$, Sakha $3\left(P_{4}\right)$ and Sakah $1\left(\mathrm{P}_{5}\right)$ at 100\% ETo during 2014/2015 and 2015/2016 seasons. A: Mesophyll thickness $(\mu \mathrm{m})$, B: Midrib thickness $(\mu \mathrm{m})($ Bar $=100 \mu \mathrm{m})$. 


\section{At $60 \%$ ET0}
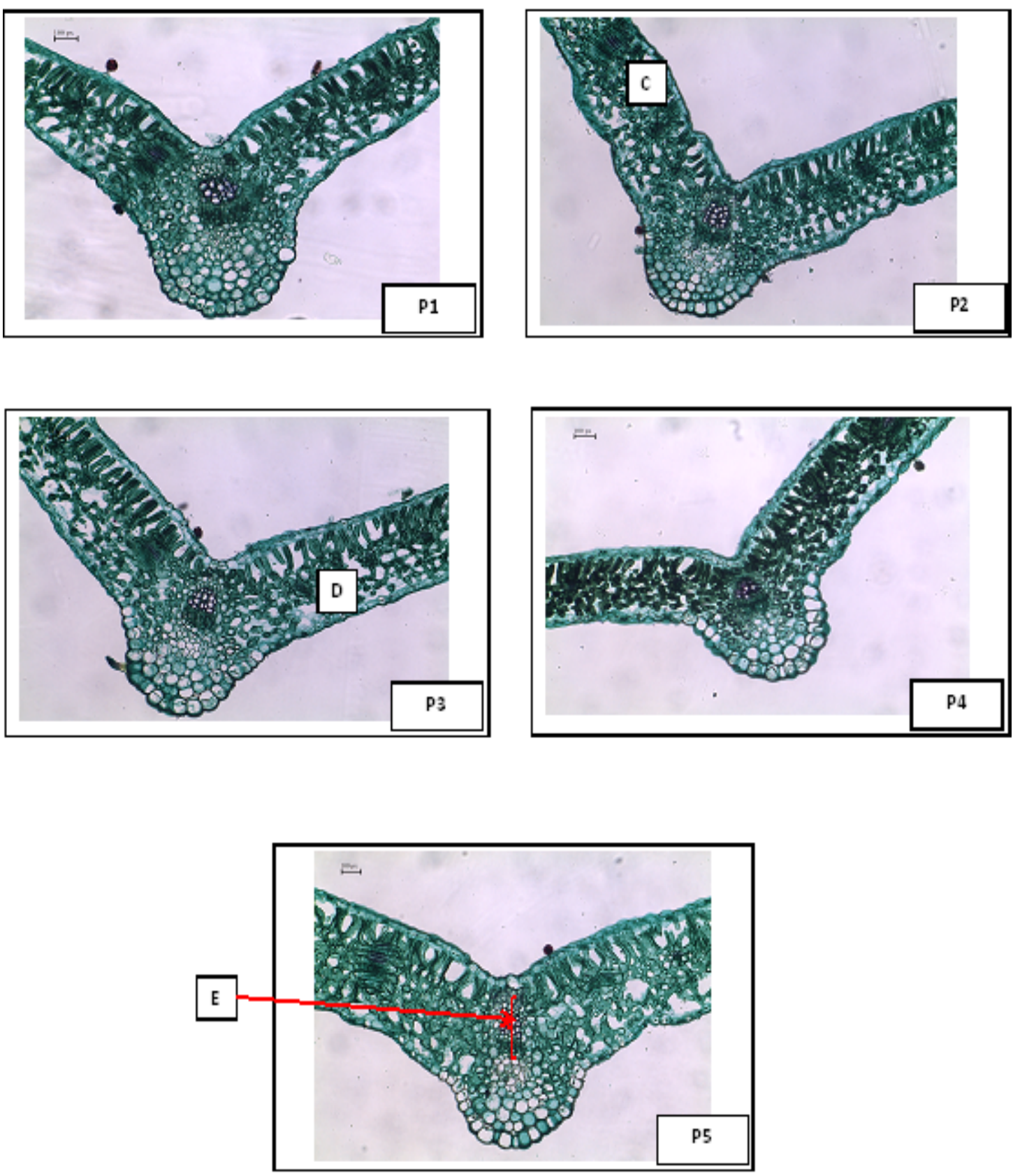

Plate 2. Cross sections of leaves of faba bean cultivars, Giza $843\left(P_{1}\right)$, Giza $3\left(P_{2}\right)$, Maser $3\left(P_{3}\right)$, Sakha $3\left(P_{4}\right)$ and Sakah $1\left(P_{5}\right)$ at 60\% ETo during 2014/2015 and 2015/2016 seasons. C: Palisade tissue ( $\left.\mu \mathrm{m}\right)$, D: Spongy tissue, E: Vascular bundle thickness $(\mu \mathrm{m})(\mathrm{Bar}=100 \mu \mathrm{m})$.

Data of crosses were presented in Table 2 and Plates 3, 4, 5 and 6. Crosses $\left(\mathrm{P}_{1} \mathrm{xP}_{2}, \mathrm{P}_{2} \mathrm{xP}_{5}\right.$, $\mathrm{P}_{1} \mathrm{xP}_{5}$ and $\mathrm{P}_{2} \mathrm{xP}_{3}$ ) appeared more tolerant under water stress, although, water deficit reduced all anatomical characters except number of xylem vessels which is the one of important character related positively with drought. Which is noteworthy that parent $\mathrm{P}_{5}$ and cross $\mathrm{P}_{3} \mathrm{xP}_{5}$ the best genotypes in number of xylem vessels. These results were agreement with Boghdady (2009) and Petrov et al. (2012). 

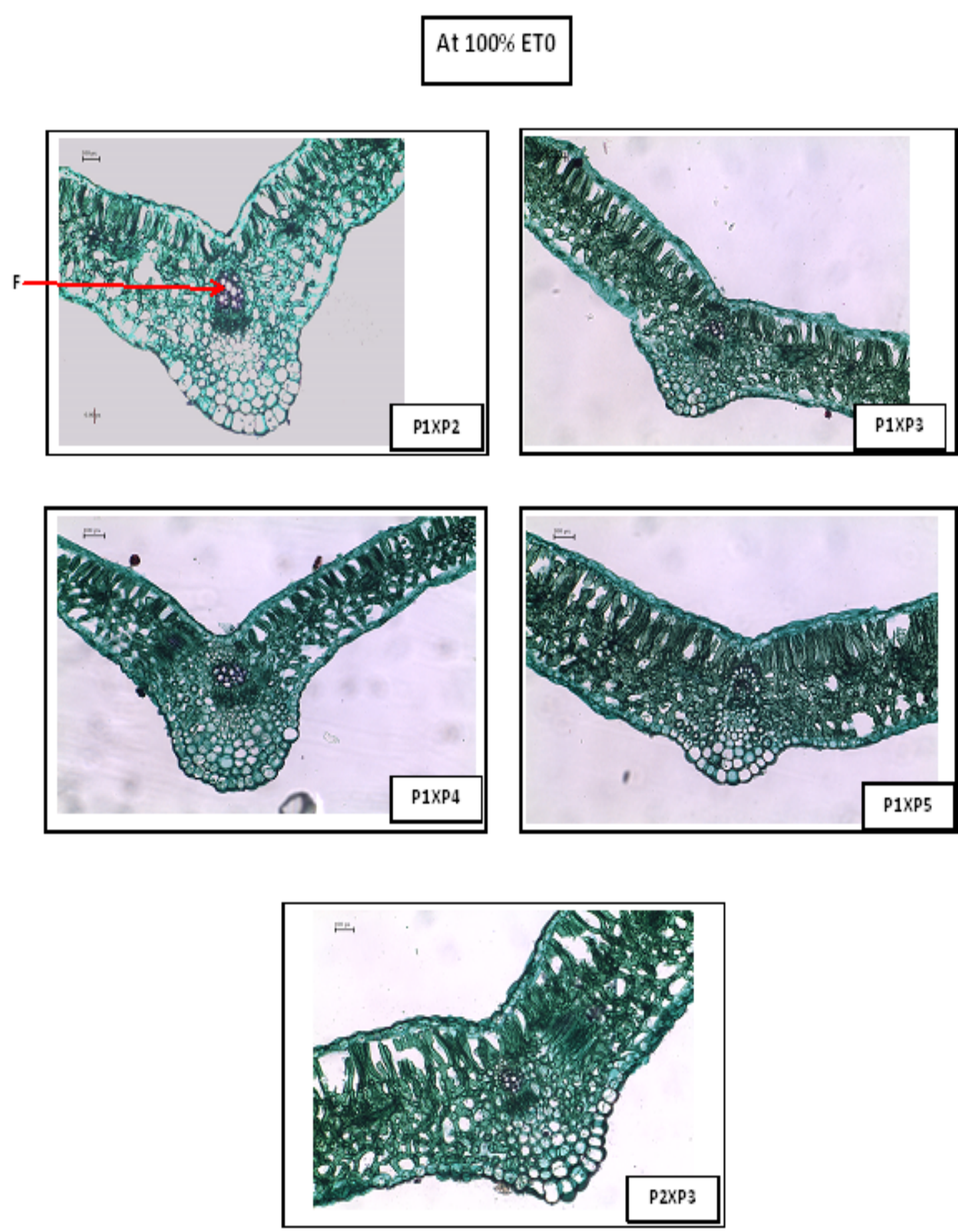

Plate 3. Cross sections of leaves of faba bean cultivars. Giza $843\left(P_{1}\right)$, Giza $3\left(P_{2}\right)$, Maser $3\left(P_{3}\right)$, Sakha $3\left(P_{4}\right)$ and Sakah $1\left(P_{5}\right)$ and their crosses $\left(10 F_{1}\right.$ hybrids) at 100\% ETo during 2014/2015 and 2015/2016 seasons. F: Xylem vessles $(B a r=100 \mu \mathrm{m})$. 

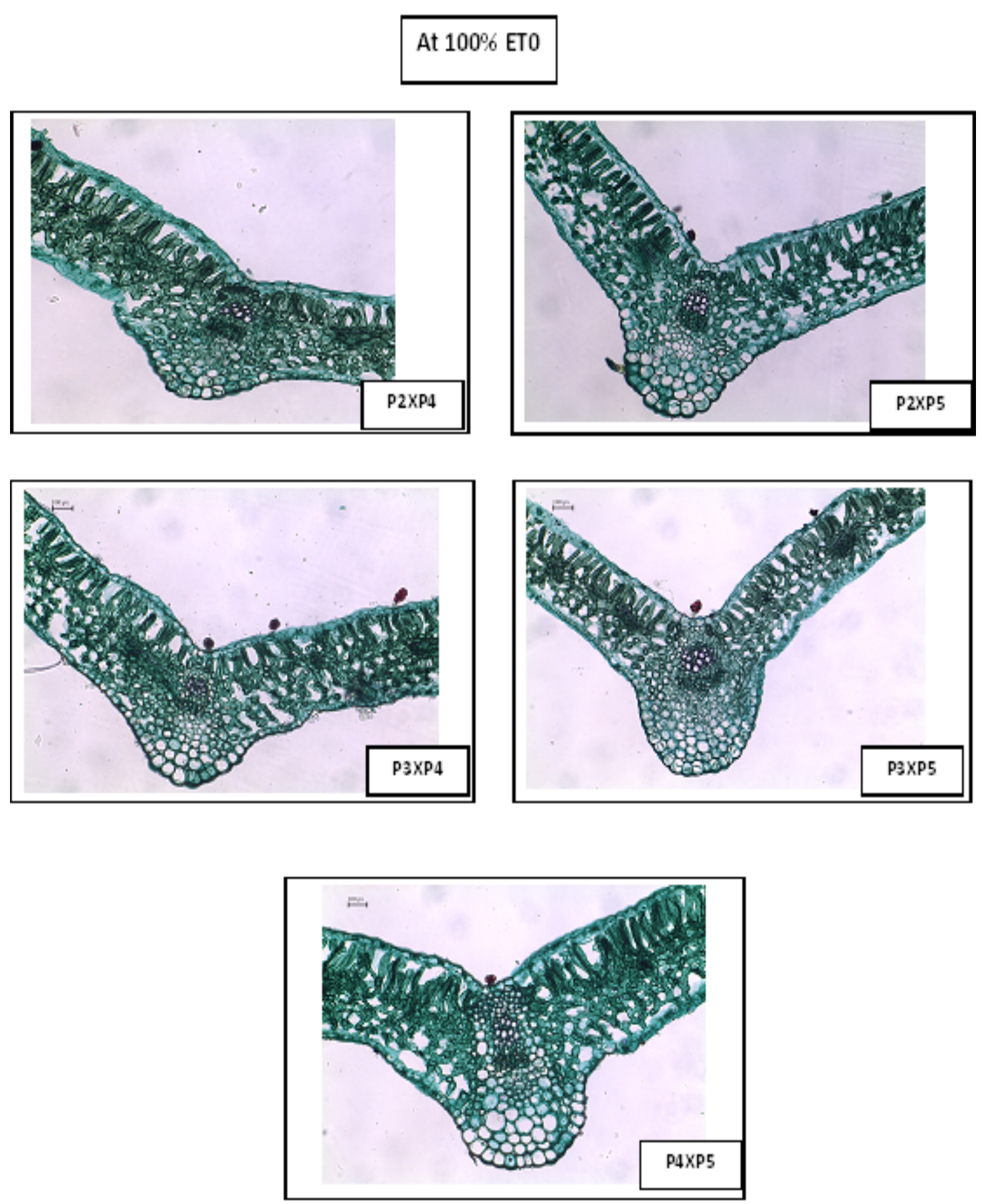

Plate 4. Cross sections of leaves of faba bean cultivars. Giza $843\left(P_{1}\right)$, Giza $3\left(P_{2}\right)$, Maser $3\left(P_{3}\right)$, Sakha $3\left(P_{4}\right)$ and Sakah $1\left(P_{5}\right)$ and their crosses $\left(10 F_{1}\right.$ hybrids) at 100\% ETo during 2014/2015 and 2015/2016 seasons. (Bar= $100 \mu \mathrm{m})$. 


\section{At $60 \%$ ETO}
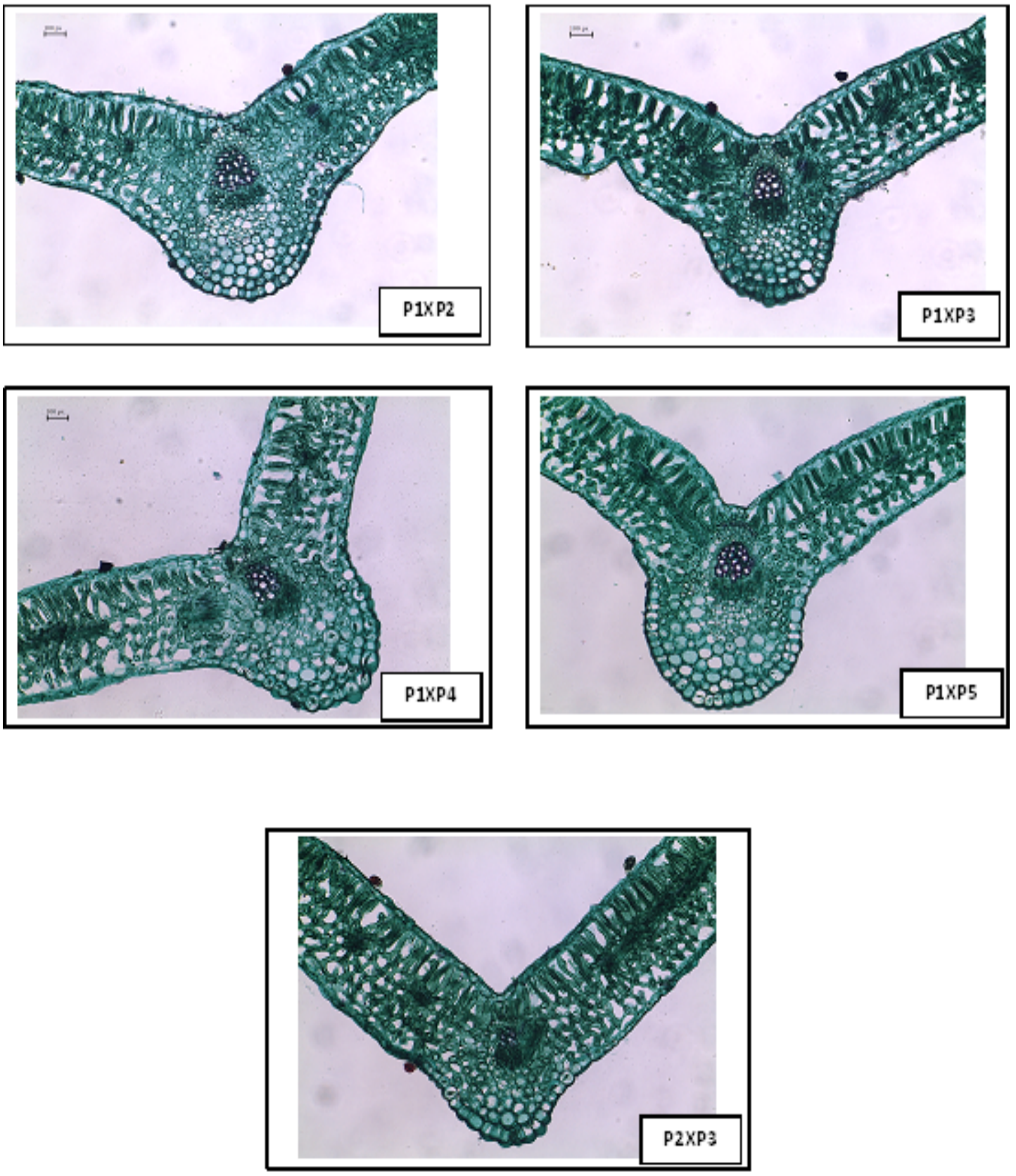

Plate 5. Cross sections of leaves of faba bean cultivars. Giza $843\left(P_{1}\right)$, Giza $3\left(P_{2}\right)$, Maser $3\left(P_{3}\right)$, Sakha $3\left(P_{4}\right)$ and Sakah $1\left(P_{5}\right)$ and their crosses $\left(10 F_{1}\right.$ hybrids) at $60 \%$ ETo during 2014/2015 and 2015/2016 seasons. (Bar= $100 \mu \mathrm{m})$. 

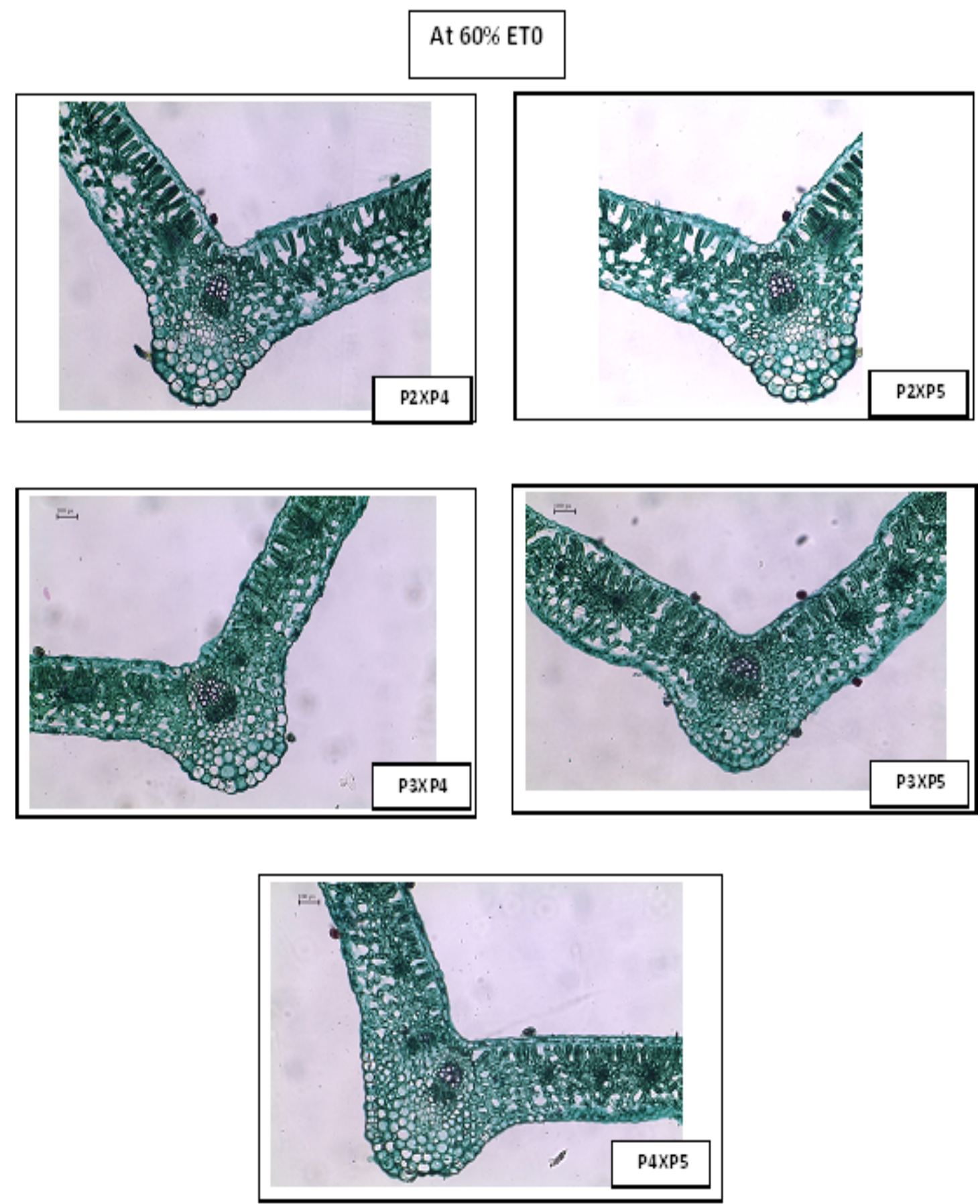

Plate 6. Cross sections of leaves of faba bean cultivars. Giza $843\left(P_{1}\right)$, Giza $3\left(P_{2}\right)$, Maser $3\left(P_{3}\right)$, Sakha $3\left(P_{4}\right)$ and Sakah $1\left(P_{5}\right)$ and their crosses $\left(10 F_{1}\right.$ hybrids) at 60\% ETo during 2014/2015 and 2015/2016 seasons. (Bar= $100 \mu \mathrm{m})$.

The results obtained by Forouzan et al. (2013) revealed the positive and significant correlations between grain yield with leaf thickness and xylem width under drought stress conditions. Due to this fact, the leaves thickness and xylem width considered key structural features of leaves that manage the ability of a safflower genotype to tolerate water deficit stress. Therefore these traits could be used as criteria to select tolerant genotype that were more tolerant to drought. 


\section{Photosynthetic pigments}

Data in Table 3 showed the values of chlorophyll a, b and carotenoids. The two parental genotypes Maser $3\left(\mathrm{P}_{3}\right)$ and Sakha $1\left(\mathrm{P}_{5}\right)$ exhibited the highest values of chlorophyll a, b $(4.4,7.8$ for $\mathrm{P}_{3}$ and 3.1, 5.4 for $\mathrm{P}_{5}$, respectively) under severe water stress. The two parental genotypes Giza $843\left(\mathrm{P}_{1}\right)$ and Sakha $3\left(\mathrm{P}_{4}\right)$ gave the highest values ofcarotenoids (2.3 and 2.5, respectively) under severe water stress. With respect to the $\mathrm{F}_{1}$ crosses, results indicated that $\mathrm{P}_{1} \mathrm{xP}_{3}$ and $\mathrm{P}_{3} \mathrm{xP}_{5}$ crosses recorded the highest of chlorophyll $\mathrm{a}, \mathrm{b}(3.4,5.8$ for $\mathrm{P}_{1} \mathrm{xP}_{3}$ and $3.2,5.6$ for $\mathrm{P}_{3} \mathrm{xP}_{5}$, respectively) under severe water stress. Concerning carotenoids, $\left(\mathrm{P}_{2} \mathrm{xP}_{4}\right)$ and $\left(\mathrm{P}_{2} \mathrm{xP}_{5}\right)$ crosses exhibited the highest values (2.8 and 2.3, respectively) under water stress. The activity of a chlorophyll degrading enzymes like chlorophyllase enzyme caused decreasing of chlorophyll content (Reddy \& Vora, 1986). These findings may suggest that these parents and $F_{1}$ crosses had high ability to drought resistance.

\section{Seed yield per plant}

Data of seed yield was presented in Table 4. Two parental genotypes; Sakha $3\left(\mathrm{P}_{4}\right)$ and Giza $843\left(\mathrm{P}_{1}\right)$ gave the highest values of seed yield/ plant (52.3 and 50.3, respectively) under severe water stress.

Comparison of performance of $\mathrm{F}_{1}$ crosses to the corresponding highest parents revealed that two crosses had the highest values of seed yield / plant namely $\mathrm{P}_{1} \mathrm{xP}_{5}$ (76.7) and $\mathrm{P}_{1} \mathrm{xP}_{4}$ (70.0) under severe water stress. These results suggest that the above mentioned parents and $F_{1}$ crosses may be of value for improving seed yield of faba bean. These superior genotypes can be exploits in breeding programs for improving drought tolerance.

\section{General combining ability and heritability estimates}

General combining ability (GCA) variances are in general attributed to the additive and additive $\mathrm{x}$ additive gene effects, while specific combining ability (SCA) variances are attributed to non-additive gene effects which involve dominance and epistasis components of genetic variation. GCA and SCA mean squares were highly significant for all measured traits under two water conditions or regimes (Tables 5, 6 and 7 ), indicating that both of them were playing an important role in the inheritance of these traits. Variances of GCA were larger than those for SCA. $\sigma^{2} \mathrm{GCA} / \sigma^{2} \mathrm{SCA}$ ratio was more than unity for number of xylem vessels, thickness of mysophyll $(\mu \mathrm{m})$, thickness of midrib $(\mu \mathrm{m})$, thickness of palisade tissue $(\mu \mathrm{m})$ and chlorophyll b under two water regimes (100\% ETo and 60\% ETo). Also, leaves number/plant, shoot fresh weight/plant $(\mathrm{g})$, root fresh weight/plant $(\mathrm{g})$, root dry weight/ plant (g), seed yield/plant (g), chlorophyll a and carotenoids under $60 \%$ ETo only, thickness of spongy tissue $(\mu \mathrm{m})$ and thickness of vascular bundle $(\mu \mathrm{m})$ under $100 \%$ ETo only. The results indicated that GCA variance was more important than SCA ones in the genetics of these traits, explaining the additive gene action in genetic makeup of traits. These finding revealed that these traits could be developed and improved through phenotypic selection. On the other hand, the variance due to SCA was more pronounced for branches number/plant and shoot dry weight/ plant (g) under two water regimes, furthermore, leaves number/plant, shoot fresh weight/plant (g), root fresh weight/plant $(\mathrm{g})$, root dry weight/ plant $(\mathrm{g})$, seed yield/plant $(\mathrm{g})$, chlorophyll a and carotenoids under $100 \%$ ETo, while thickness of vascular bundle $(\mathrm{mm})$ under $60 \%$ ETo. From these results, it could be concluded that the controlling of the inheritance processes for the measurements characters depend on additive and dominance effects of genetic components, although there were varied contribution of each component according to measurement and water regime (Ahmad \& Hager, 2010; Haridy \& Amein, 2011; Farag \& Afiah, 2012; Hazem et al., 2013; Ashrei et al., 2014; Zeinab \& Helal, 2014; Bishnoi, 2016; Ismail, 2016; Abdalla et al., 2017 and Bishnoi et al., 2018a).

The results in Table 5 showed that the values of heritability in narrow sense were ranged from 5.92 to $58.6 \%$ for growth characters and ranged from 21.3 to $57.5 \%$ for anatomical characters (Table 6) under two water regimes. Respecting to photosynthetic pigments characters (Table 7), the values of heritability in narrow sense were ranged from 1.68 to $57.5 \%$ under two water regimes. With respect to seed yield per plant were 7.5 and 45.8 under two water regimes. These results indicated that these characters are greatly influenced by dominance and environmental conditions. Similar findings were obtained by Abdelmula et al. (1999), Ibrahim (2010), El-Bramawy \& Osman (2012), Peyman (2015), Abd El-Zaher (2016) and Ismail (2016). 


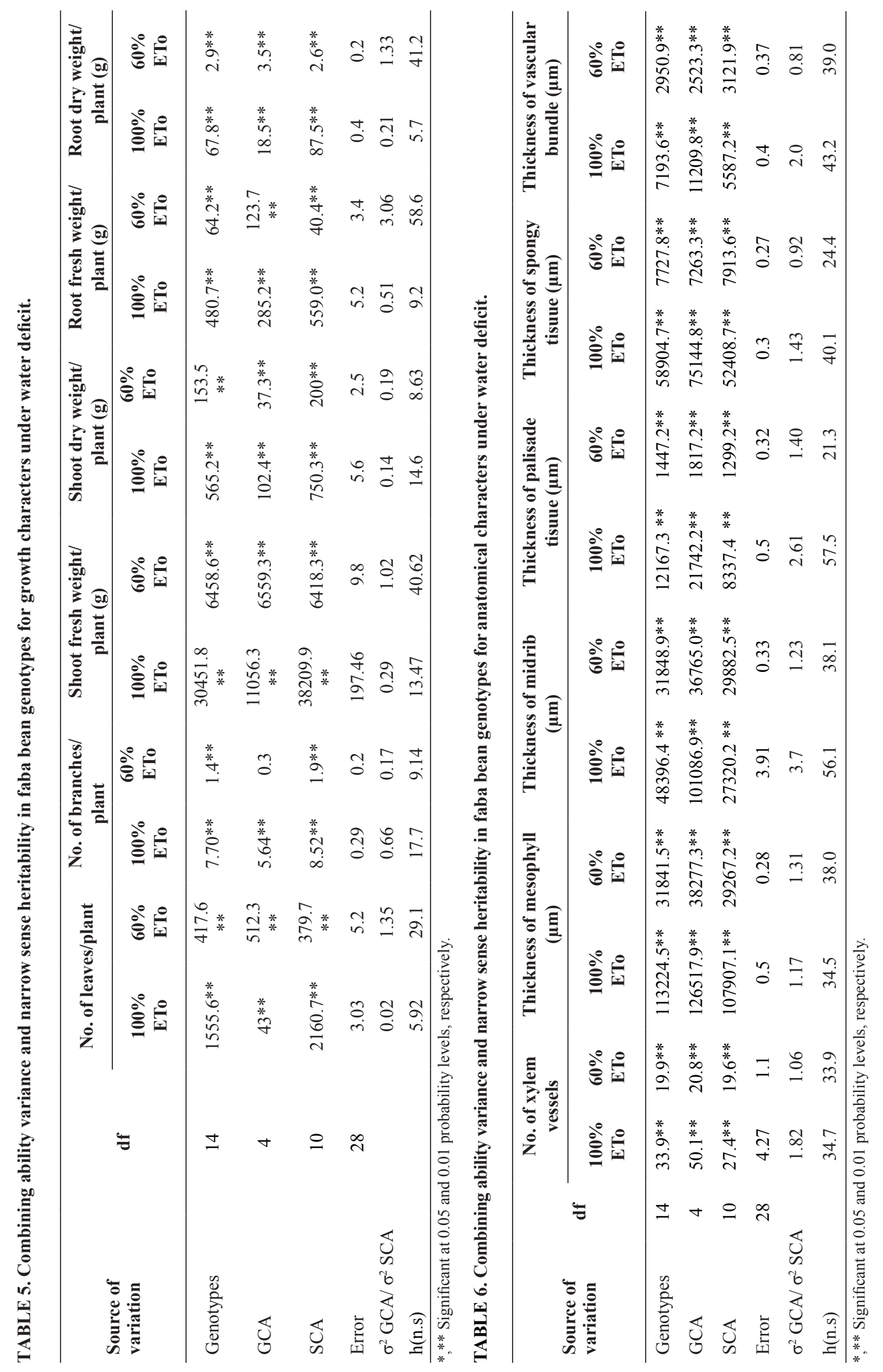

Egypt. J. Agron. 40, No.3 (2018) 
TABLE 7. Combining ability variance and narrow sense heritability in faba bean genotypes for seed yield/plant (g), chlorophyll $a, b$ and carotenoids under water deficit.

\begin{tabular}{lccccccccc}
\hline \multirow{2}{*}{$\begin{array}{l}\text { Source of } \\
\text { variation }\end{array}$} & df & \multicolumn{2}{c}{ Seed yield/plant $(\mathbf{g})$} & \multicolumn{2}{c}{ Chlorophyll a } & \multicolumn{2}{c}{ Chlorophyll b } & \multicolumn{2}{c}{ Carotenoids } \\
\cline { 2 - 10 } & & $\begin{array}{c}\mathbf{1 0 0 \%} \\
\text { ETo }\end{array}$ & $\begin{array}{c}\mathbf{6 0 \%} \\
\text { ETo }\end{array}$ & $\begin{array}{c}\mathbf{1 0 0 \%} \\
\text { ETo }\end{array}$ & $\begin{array}{c}\mathbf{6 0 \%} \\
\text { ETo }\end{array}$ & $\begin{array}{c}\mathbf{1 0 0 \%} \\
\text { ETo }\end{array}$ & $\begin{array}{c}\mathbf{6 0 \%} \\
\text { ETo }\end{array}$ & $\begin{array}{c}\mathbf{1 0 0 \%} \\
\text { ETo }\end{array}$ & $\begin{array}{c}\mathbf{6 0 \%} \\
\text { ETo }\end{array}$ \\
\hline Genotypes & 4 & $3351.4^{* *}$ & $600.7^{* *}$ & $2.4^{* *}$ & $2.0^{* *}$ & $5.9^{* *}$ & $5.8^{* *}$ & $0.9^{* *}$ & $0.61^{* *}$ \\
GCA & 4 & $804.9 * *$ & $705.7^{* *}$ & $2.2^{* *}$ & $4.0^{* *}$ & $9.4^{* *}$ & $11.9^{* *}$ & $0.1^{* *}$ & $0.86^{* *}$ \\
$\mathrm{SCA}$ & 10 & $4370.0^{* *}$ & $558.7^{* *}$ & $2.5^{* *}$ & $1.2^{* *}$ & $4.5^{* *}$ & $3.4^{* *}$ & $1.2^{* *}$ & $0.51^{* *}$ \\
Error & 28 & 16.0 & 1.99 & 0.01 & 0.01 & 0.01 & 0.01 & 0.01 & 0.01 \\
$\sigma^{2} \mathrm{GCA} / \sigma^{2} \mathrm{SCA}$ & & 0.18 & 1.26 & 0.89 & 3.30 & 2.1 & 3.53 & 0.05 & 1.70 \\
$\mathrm{~h}(\mathrm{n} . \mathrm{s})$ & & 7.5 & 45.8 & 23.3 & 56.8 & 41.6 & 57.5 & 1.68 & 41.9 \\
\hline
\end{tabular}

*** Significant at 0.05 and 0.01 probability levels, respectively.

The general combining ability effects of parents are presented in Tables 8, 9 and 10 . Result revealed that the parent P5 (Sakha 1) showed positive and highly significant effects for thickness of mysophyll $(\mu \mathrm{m})$, thickness of midrib $(\mu \mathrm{m})$, thickness of vascular bundle $(\mu \mathrm{m})$, chlorophyll a and chlorophyll $b$ under two water regimes, moreover it showed highly significant positive for shoot dry weight/plant $(\mathrm{g})$, number of xylem vessels and carotenoids under 100\% ETo only and for thickness of palisade tissue $(\mu \mathrm{m})$, thickness of spongy tissue $(\mu \mathrm{m})$ and seed yield/ plant (g) under $60 \%$ ETo only. $\mathrm{P}_{2}$ (Giza 3 ) revealed good combiner for root fresh weight/ plant $(\mathrm{g})$, root dry weight/plant $(\mathrm{g})$, thickness of mysophyll $(\mu \mathrm{m})$ and thickness of spongy tissue $(\mu \mathrm{m})$ under two water regimes, furthermore it showed highly significant positive for shoot fresh weight/plant (g), number of xylem vessels and thickness of midrib $(\mu \mathrm{m})$ under $60 \%$ ETo only and for thickness of palisade tissue $(\mu \mathrm{m})$ and thickness of vascular bundle $(\mu \mathrm{m})$ under $100 \%$ ETo only. $\mathrm{P}_{4}$ (Sakha 3) demonstrated highly significant positive effects for seed yield/plant (g), thickness of midrib $(\mu \mathrm{m})$ and branches number/plant under two water regimes; highly significant positive for leaves number/plant, thickness of palisade tissue $(\mu \mathrm{m})$, thickness of spongy tissue $(\mu \mathrm{m})$, thickness of vascular bundle $(\mu \mathrm{m})$ and chlorophyll $\mathrm{b}$ under $100 \%$ ETo only and for carotenoids under $60 \%$ ETo only. $\mathrm{P}_{1}$ (Giza 843) showed highly positive significant effects for root fresh weight/plant (g) and seed yield/plant (g) under two water regimes, moreover it showed highly significant positive for leaves number/plant, shoot fresh weight/plant (g), shoot dry weight/plant (g), number of xylem vessels, thickness of midrib $(\mu \mathrm{m})$, thickness of vascular bundle $(\mu \mathrm{m})$ and carotenoids under $60 \%$ ETo while root dry weight/plant (g) under 100\% ETo. $\mathrm{P}_{3}$ (Maser 3) exhibited highly significant positive effects for chlorophyll a under two water regimes, furthermore it showed highly significant positive for seed yield/plant (g)and growth characters except for root dry weight/plant $(\mathrm{g})$ under $100 \%$ ETo only and for chlorophyll $\mathrm{b}$ and thickness of spongy tissue $(\mu \mathrm{m})$ under $60 \%$ ETo only. Preceding parents are studied to be good general combiners for their particular traits. Also results recommend that three parental genotypes; $\mathrm{P}_{5}$ (Sakha 1), $\mathrm{P}_{2}$ (Giza 3 ) and $\mathrm{P}_{4}$ (Sakha 3 ) are a good combiners for improving most characters. These results were in harmony by Alghamdi (2007), Alghamdi (2009), Farag \& Afiah (2012), Zeinab \& Helal (2014), Bishnoi (2016), Abdalla et al. (2017) and Bishnoi et al. (2018b).

\section{Specific combining ability estimates}

Estimates of SCA effects for $\mathrm{F}_{1}$ crosses were presented in Tables 11, 12 and 13. Three crosses $\left(\mathrm{P}_{3} \mathrm{xP}_{4}, \mathrm{P}_{3} \mathrm{xP}_{5}\right.$ and $\left.\mathrm{P}_{1} \mathrm{xP}_{5}\right)$ had highly positive significant SCA effects for most growth characters under two water regimes, while three crosses $\left(\mathrm{P}_{1} \mathrm{xP}_{2}, \mathrm{P}_{1} \mathrm{xP}_{3}\right.$ and $\left.\mathrm{P}_{2} \mathrm{xP}_{3}\right)$ appeared highly positive significant SCA effects for most anatomical characters under two water regimes. Regarding photosynthetic pigments characters and seed yield/plant (g), crosses $\mathrm{P}_{1} \mathrm{xP}_{5}, \mathrm{P}_{2} \mathrm{x}_{4}$ and $\mathrm{P}_{2} \mathrm{xP}_{5}$ exhibited highly significant and positive $\mathrm{SCA}^{2}$ effects under two water regimes for most of these characters. The superiority crosses which demonstrated desirable SCA effects showed high heterosis values for characters. These superiority hybrids could be used in breeding programs to produce high yielding ability and drought tolerance pure lines of faba bean. These results are in line with those reported by Alghamdi (2009), Ibrhim (2010), Farag \& Afiah (2012) and Abdalla et al. (2017), who found the crosses included high and low combiners had best SCA effects. 
TABLE 8. General combining ability effects for growth charactersunder water deficit.

\begin{tabular}{|c|c|c|c|c|c|c|c|c|c|c|c|c|}
\hline \multirow{2}{*}{ Genotypes } & \multicolumn{2}{|c|}{$\begin{array}{c}\text { No. of leaves/ } \\
\text { plant }\end{array}$} & \multicolumn{2}{|c|}{$\begin{array}{c}\text { No. of branches/ } \\
\text { plant }\end{array}$} & \multicolumn{2}{|c|}{$\begin{array}{l}\text { Shoot fresh } \\
\text { weight (g) }\end{array}$} & \multicolumn{2}{|c|}{$\begin{array}{l}\text { Shoot dry } \\
\text { weight (g) }\end{array}$} & \multicolumn{2}{|c|}{$\begin{array}{l}\text { Root fresh } \\
\text { weight (g) }\end{array}$} & \multicolumn{2}{|c|}{$\begin{array}{c}\text { Root dry weight } \\
\text { (g) }\end{array}$} \\
\hline & $\begin{array}{c}100 \% \\
\text { ETo }\end{array}$ & $\begin{array}{l}60 \% \\
\text { ETo }\end{array}$ & $\begin{array}{c}100 \% \\
\text { ETo }\end{array}$ & $\begin{array}{l}60 \% \\
\text { ETo }\end{array}$ & $\begin{array}{c}100 \% \\
\text { ETo }\end{array}$ & $\begin{array}{l}60 \% \\
\text { ETo }\end{array}$ & $\begin{array}{c}100 \% \\
\text { ETo }\end{array}$ & $\begin{array}{l}60 \% \\
\text { ETo }\end{array}$ & $\begin{array}{c}100 \% \\
\text { ETo }\end{array}$ & $\begin{array}{l}60 \% \\
\text { ETo }\end{array}$ & $\begin{array}{c}100 \% \\
\text { ETo }\end{array}$ & $\begin{array}{l}60 \% \\
\text { ETo }\end{array}$ \\
\hline $\mathrm{P}_{1}$ & 0.11 & $8.5^{* *}$ & $-0.24^{*}$ & -0.14 & 1.0 & $23^{* *}$ & 0.1 & $1.8^{* *}$ & $2.1 * *$ & $0.9 * *$ & $1.2 * *$ & 0.13 \\
\hline $\mathrm{P}_{2}$ & $-1.4^{* *}$ & $-4.1 * *$ & $-0.3 * *$ & 0.00 & $-7.5^{* *}$ & $8.3 * *$ & $-2.3 * *$ & 0.4 & $3.9 * *$ & $3.2 * *$ & $0.4 * *$ & $0.51 * *$ \\
\hline $\mathrm{P}_{3}$ & $0.83^{*}$ & $-1.2^{* *}$ & $0.76^{* *}$ & 0.00 & $38.0^{* *}$ & $-4.4^{* *}$ & $2.7 * *$ & 0.3 & $1.8^{* *}$ & 0.7 & 0.2 & 0.18 \\
\hline $\mathrm{P}_{4}$ & $1.88 * *$ & $-2.3 * *$ & $0.29 * *$ & $0.19^{*}$ & $-23 * *$ & $-1.7^{* *}$ & $-2.1 * *$ & $-1.6^{* *}$ & $-4.4 * *$ & $-2.2 * *$ & $-1.2 * *$ & $-0.30 * *$ \\
\hline $\mathrm{P}_{5}$ & $-1.4 * *$ & $-0.9^{*}$ & $-0.5 * *$ & -0.05 & $-8.2 * *$ & $-25 * *$ & $1.5^{* *}$ & $-0.9 * *$ & $-3.5 * *$ & $-2.6^{* *}$ & $-0.5 * *$ & $-0.51^{* *}$ \\
\hline S.E.(gi-gj) & 0.34 & 0.45 & 0.106 & 0.086 & 2.74 & 0.611 & 0.46 & 0.309 & 0.44 & 0.36 & 0.2 & 0.1 \\
\hline
\end{tabular}

*,** Significant at 0.05 and 0.01 probability levels, respectively.

TABLE 9. General combining ability effects foranatomical characters under water deficit.

\begin{tabular}{|c|c|c|c|c|c|c|c|c|c|c|c|c|}
\hline Genotypes & \multicolumn{2}{|c|}{$\begin{array}{l}\text { No. of xylem } \\
\text { vessels }\end{array}$} & \multicolumn{2}{|c|}{$\begin{array}{c}\text { Thickness of } \\
\text { mesophyll ( } \mu \mathrm{m})\end{array}$} & \multicolumn{2}{|c|}{$\begin{array}{l}\text { Thickness of } \\
\text { midrib }(\mu \mathrm{m})\end{array}$} & \multicolumn{2}{|c|}{$\begin{array}{c}\text { Thickness of } \\
\text { palisade tisuue } \\
(\mu \mathrm{m})\end{array}$} & \multicolumn{2}{|c|}{$\begin{array}{c}\text { Thickness of } \\
\text { spongy tisuue } \\
(\mu \mathrm{m})\end{array}$} & \multicolumn{2}{|c|}{$\begin{array}{c}\text { Thickness } \\
\text { of vascular } \\
\text { bundle }(\mu \mathrm{m})\end{array}$} \\
\hline $\mathrm{P}_{1}$ & $-1.7 * *$ & $1.4 * *$ & $-58.3 * *$ & $-26.5 * *$ & $-94.7 * *$ & $10.2^{* *}$ & $-39.0 * *$ & $-2.8 * *$ & $-16.1 * *$ & $-21.6 * *$ & -0.1 & $17.9 * *$ \\
\hline $\mathrm{P}_{3}$ & 0.52 & $-0.5^{*}$ & $-90.7 * *$ & $-19.3 * *$ & $-24.7 * *$ & $-69.1 * *$ & -0.2 & $-1.0 * *$ & $-61.0 * *$ & $6.1 * *$ & $-39.5 * *$ & $-7.9 * *$ \\
\hline $\mathrm{P}_{4}$ & -0.24 & $-1.2 * *$ & $-3.3 * *$ & $-45.0 * *$ & $41.6^{* *}$ & $4.3 * *$ & $21.1 * *$ & $-6.2 * *$ & $8.2 * *$ & $-16.2 * *$ & $17.7 * *$ & $-8.9 * *$ \\
\hline $\mathrm{P}_{5}$ & $2.33^{* *}$ & -0.2 & $55.3 * *$ & $51.1 * *$ & $88.5^{* *}$ & $44.9 * *$ & $-22.7 * *$ & $16.2 * *$ & $-28.3^{* *}$ & $8.4^{* *}$ & $7.2 * *$ & $2.5^{* *}$ \\
\hline
\end{tabular}

*,** Significant at 0.05 and 0.01 probability levels, respectively.

Egypt. J. Agron. 40, No.3 (2018) 
TABLE 10. General combining ability effects for seed yield/plant (g), chlorophyll a, b and carotenoids under water deficit.

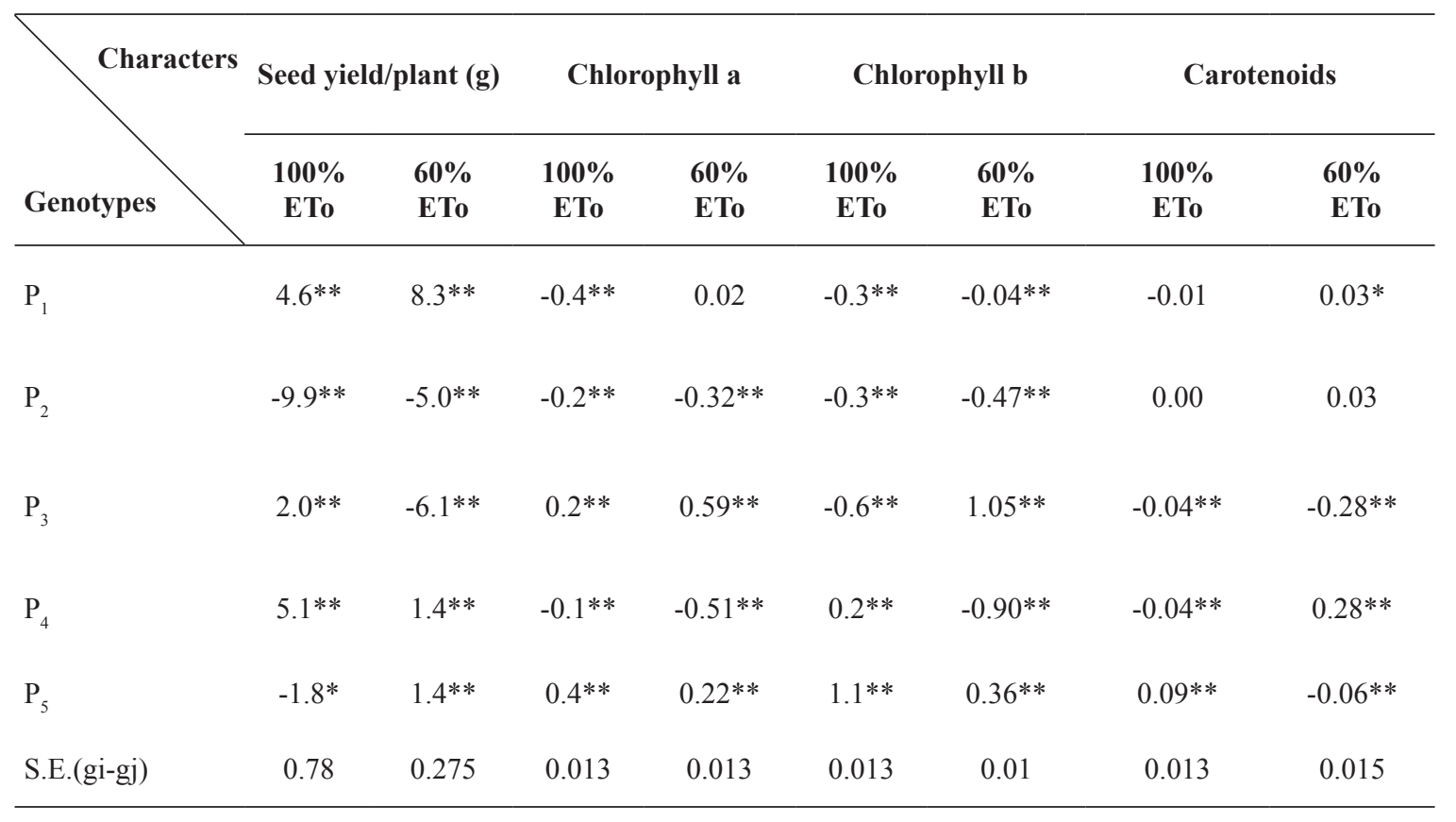

TABLE 11. Specific combining ability effects for growth characters under water deficit.

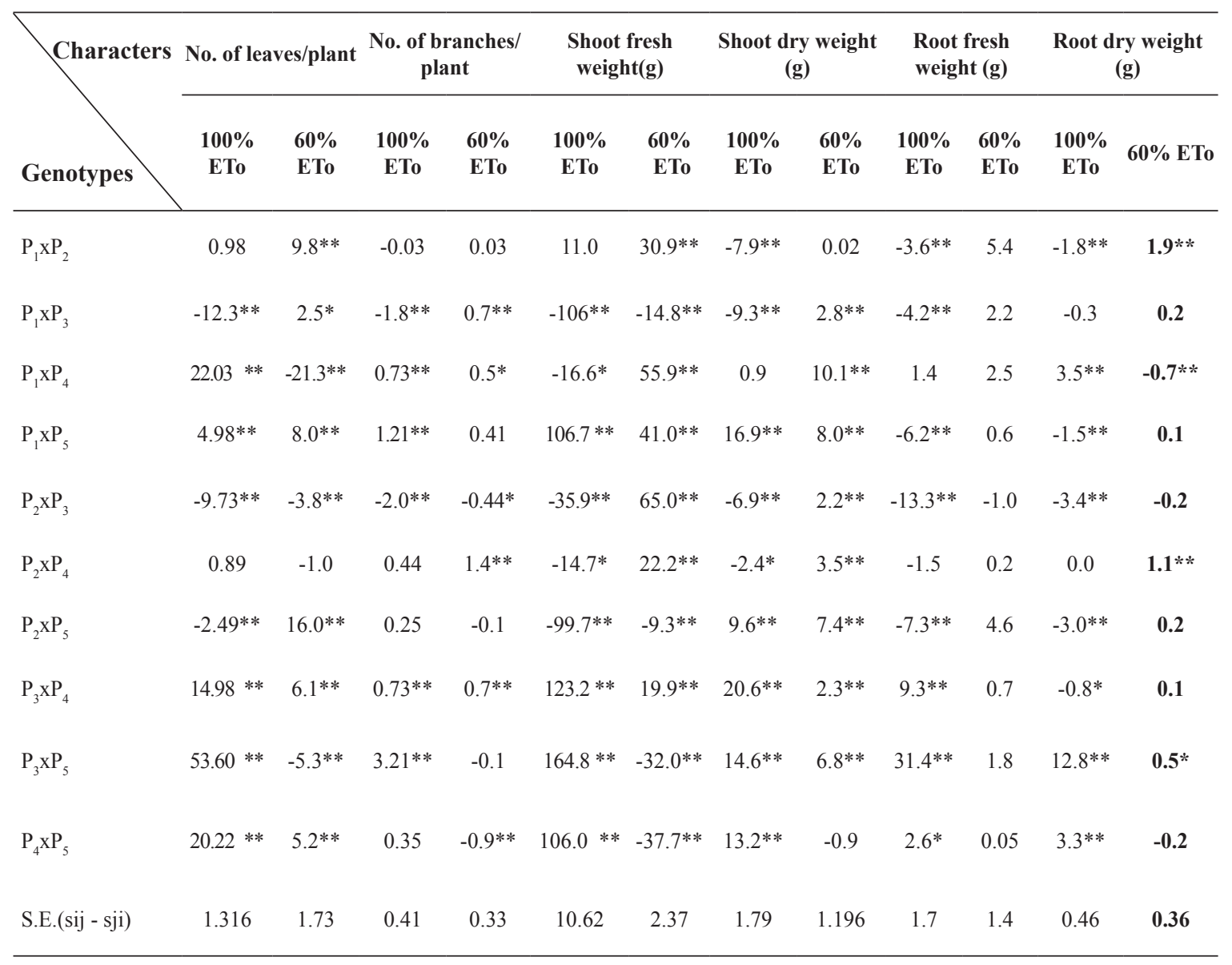

*** Significant at 0.05 and 0.01 probability levels, respectively. 


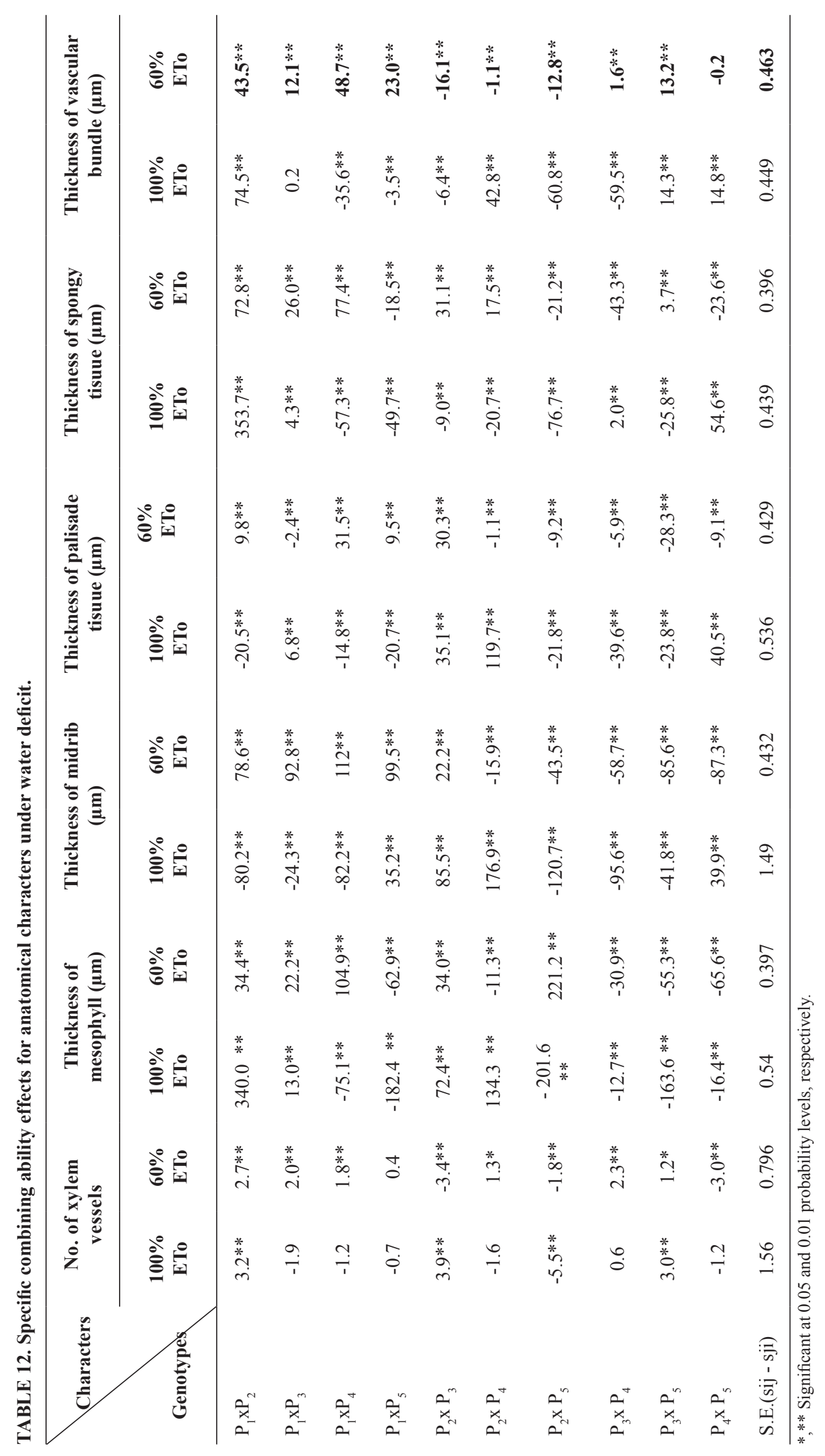

Egypt. J. Agron. 40, No.3 (2018) 
TABLE 13. Specific combining ability effects for seed yield/plant (g), chlorophyll a, $b$ and carotenoids under water deficit.

\begin{tabular}{|c|c|c|c|c|c|c|c|c|}
\hline \multirow[b]{2}{*}{ Genotypes } & \multicolumn{2}{|c|}{$\begin{array}{l}\text { Seed yield/ plant } \\
\text { (g) }\end{array}$} & \multicolumn{2}{|c|}{ Chlorophyll a } & \multicolumn{2}{|c|}{ Chlorophyll b } & \multicolumn{2}{|c|}{ Carotenoids } \\
\hline & $\begin{array}{l}100 \% \\
\text { ETo }\end{array}$ & $\begin{array}{l}60 \% \\
\text { ETo }\end{array}$ & $\begin{array}{c}100 \% \\
\text { ETo }\end{array}$ & $\begin{array}{l}60 \% \\
\text { ETo }\end{array}$ & $\begin{array}{c}100 \% \\
\text { ETo }\end{array}$ & $\begin{array}{l}60 \% \\
\text { ETo }\end{array}$ & $\begin{array}{c}100 \% \\
\text { ETo }\end{array}$ & $\begin{array}{l}60 \% \\
\text { ETo }\end{array}$ \\
\hline $\mathrm{P}_{1} \mathrm{xP}_{2}$ & $-35.3 * *$ & $-8.4 * *$ & $-0.7 * *$ & -0.04 & $-1.2 * *$ & $-0.1 * *$ & $0.30 * *$ & -0.07 \\
\hline $\mathrm{P}_{1} \mathrm{xP}_{3}$ & $-27.9^{* *}$ & $2.0 * *$ & $-0.7^{* *}$ & $0.29 * *$ & $-0.2 * *$ & $0.4^{* *}$ & $0.93 * *$ & $-0.17 * *$ \\
\hline $\mathrm{P}_{1} \mathrm{xP}_{4}$ & $16.4 * *$ & $13.2 * *$ & $0.3^{* *}$ & $-0.72 * *$ & $0.2 * *$ & $-1.2 * *$ & $-0.46 * *$ & $-0.34 * *$ \\
\hline $\mathrm{P}_{1} \mathrm{xP}_{5}$ & $73.3 * *$ & $19.9 * *$ & $0.9^{* *}$ & $0.35^{* *}$ & $1.3^{* *}$ & $0.6^{* *}$ & $-1.19 * *$ & $-0.29 * *$ \\
\hline $\mathrm{P}_{2} \mathrm{xP}_{3}$ & $4.0^{*}$ & $-9.0 * *$ & $0.5^{* *}$ & $-0.98 * *$ & $1.2^{* *}$ & $-1.7 * *$ & $-0.87 * *$ & $-0.18 * *$ \\
\hline $\mathrm{P}_{2} \mathrm{xP}_{4}$ & $25.9 * *$ & $-17.2^{* *}$ & $1.3^{* *}$ & $0.13 * *$ & $2.6^{* *}$ & $0.4 * *$ & 0.02 & $0.67 * *$ \\
\hline $\mathrm{P}_{2} \mathrm{xP}_{5}$ & $30.2 * *$ & $9.1 * *$ & $0.5^{* *}$ & $-0.59 * *$ & $-0.4 * *$ & $-0.6 * *$ & $0.40 * *$ & $0.48 * *$ \\
\hline $\mathrm{P}_{3} \mathrm{xP}_{4}$ & $-11.0 * *$ & 0.2 & $-1.0 * *$ & $-0.67 * *$ & $-1.0 * *$ & $-1.1 * *$ & $-0.44 * *$ & $-0.41 * *$ \\
\hline $\mathrm{P}_{3} \mathrm{xP}_{5}$ & $30.9 * *$ & $8.6^{* *}$ & $-0.9 * *$ & $-0.10 * *$ & $-0.6^{* *}$ & $-0.2 * *$ & $0.25^{* *}$ & $0.25^{* *}$ \\
\hline $\mathrm{P}_{4} \mathrm{xP}_{5}$ & $-13.8^{* *}$ & -1.0 & $0.1^{* *}$ & 0.01 & $0.1^{*}$ & $-0.3 * *$ & $0.14^{* *}$ & $-0.14 * *$ \\
\hline S.E.(sij - sji) & 3.02 & 1.1 & 0.05 & 0.052 & 0.05 & 0.033 & 0.05 & 0.059 \\
\hline
\end{tabular}

*** Significant at 0.05 and 0.01 probability levels, respectively.

\section{Heterosis estimate}

Tables 14, 15 and 16 were presented the heterosis values relative to mid parents (MP). For growth characters, heterosis percentages were highly significant and positive in two crosses; $\mathrm{P}_{1} \mathrm{xP}_{5}$ and $\mathrm{P}_{3} \times \mathrm{P}_{4}$ under the two water regimes. Regarding anatomical characters, the two crosses; $\mathrm{P}_{1} \times \mathrm{P}_{2}$ and $\mathrm{P}_{2} \mathrm{xP}_{4}$ exhibited highly significant and positive heterotic effects under the two water regimes. With respect to photosynthetic pigments characters and seed yield/plant $(\mathrm{g})$, crosses $\mathrm{P}_{2} \mathrm{xP}_{5}$ and $\mathrm{P}_{1} \mathrm{xP}_{5}$ (except for carotenoids) exhibited highly significant and positive heterotic effects under two water regimes. Hayman (1958) showed that the difference in values of heterosis resulted from diversity among parents with non-allelic interactions. While, Alghamdi (2009) demonstrated the heterosis estimates for most characters was appeared sufficient genetic variability among parents. Moreover,
F1 superiority in some traits showed different degrees of gene action in parental combinations that may effect by direct or indirect action on characters (Abdelmula et al., 1999; Omar, 2004; Darwish et al., 2005; Attia \& Salem, 2006; El-Hady et al., 2006; Ahmad \& Hager, 2010; Link et al., 2010; Ibrahim, 2015; Abd El-Zaher, 2016; Bishnoi, 2016 and Abdalla et al., 2017).

\section{Conclusion}

Three parental genotypes; $\mathrm{P}_{5}$ (Sakha 1), $\mathrm{P}_{2}$ (Giza 3) and $\mathrm{P}_{4}$ (Sakha 3) were considered to be good combiners. The crosses; $\mathrm{P}_{1} \mathrm{xP}_{5}$ (for most of characters), $\mathrm{P}_{3} \mathrm{xP}_{4}$ (for growth characters), $\mathrm{P}_{1} \mathrm{xP}_{2}$ (for anatomical characters) and $\mathrm{P}_{2} \mathrm{x}_{5}$ (for photosynthetic pigments characters and seed yield/plant) exhibited desirable SCA effects and significant heterosis values under two water regimes. All these parents and hybrids could be used in breeding programs for improving drought tolerance. 


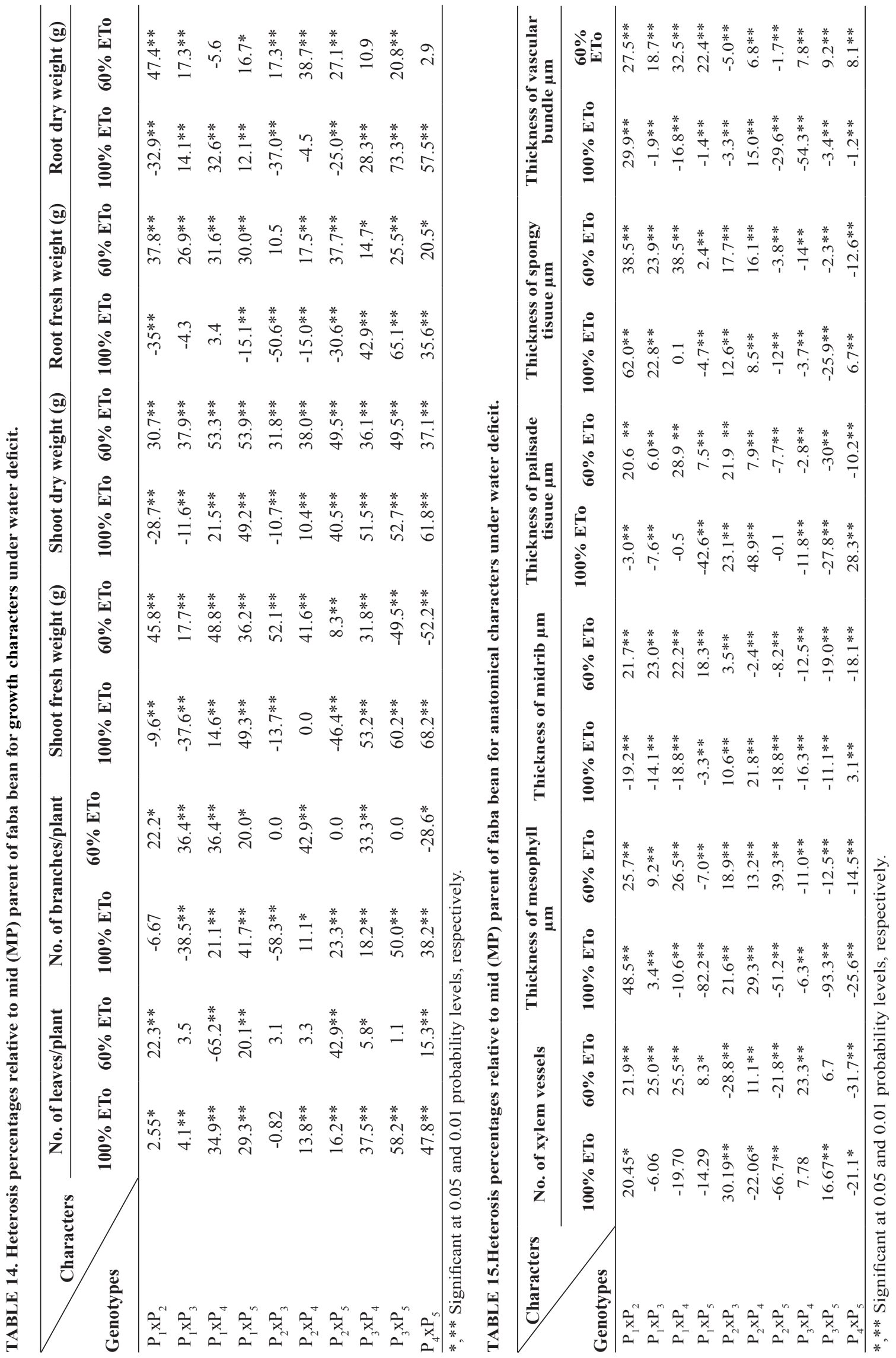

Egypt. J. Agron. 40, No.3 (2018) 
TABLE 16. Heterosis percentages relative to mid (MP) parent of faba beanfor seed yield/plant (g), chlorophyll a, $b$ and carotenoids under water deficit.

\begin{tabular}{|c|c|c|c|c|c|c|c|c|}
\hline \multirow{2}{*}{ Genaracters } & \multicolumn{2}{|c|}{ Seed yield/ plant (g) } & \multicolumn{2}{|c|}{ Chlorophyll a } & \multicolumn{2}{|c|}{ Chlorophyll b } & \multicolumn{2}{|c|}{ Carotenoids } \\
\hline & $\begin{array}{c}100 \% \\
\text { ETo }\end{array}$ & $\begin{array}{l}60 \% \\
\text { ETo }\end{array}$ & $\begin{array}{c}100 \% \\
\text { ETo }\end{array}$ & $\begin{array}{l}60 \% \\
\text { ETo }\end{array}$ & $\begin{array}{c}100 \% \\
\text { ETo }\end{array}$ & $\begin{array}{l}60 \% \\
\text { ETo }\end{array}$ & $\begin{array}{c}100 \% \\
\text { ETo }\end{array}$ & $\begin{array}{l}60 \% \\
\text { ETo }\end{array}$ \\
\hline $\mathrm{P}_{1} \mathrm{xP}_{2}$ & $-35.5^{* *}$ & $-19.4 * *$ & $-29.7 * *$ & $-20.0 * *$ & $-27.1 * *$ & $-21.0^{* *}$ & $9.9 * *$ & -3.1 \\
\hline $\mathrm{P}_{1} \times \mathrm{P}_{3}$ & $-27.0 * *$ & $17.9 * *$ & $-76.3 * *$ & $-3.2 * *$ & $-10.9 * *$ & $-7.0^{* *}$ & $36.1 * *$ & $-37.1 * *$ \\
\hline $\mathrm{P}_{1} \mathrm{xP}_{4}$ & $21.0 * *$ & $26.7 * *$ & $14.3 * *$ & $-81.2 * *$ & $14.6^{* *}$ & $-84.9 * *$ & $-92.2 * *$ & $-34.0 * *$ \\
\hline $\mathrm{P}_{1} \times \mathrm{P}_{5}$ & $61.1 * *$ & $46.5 * *$ & $25.4 * *$ & $7.5^{* *}$ & $22.3 * *$ & $6.7 * *$ & $-676.2 * *$ & $-28.7 * *$ \\
\hline $\mathrm{P}_{2} \mathrm{xP}_{3}$ & $9.2 * *$ & $-55.6^{* *}$ & $9.3^{* *}$ & $-95.5^{* *}$ & $34.2 * *$ & $-91.0 * *$ & $-223.0 * *$ & -5.5 \\
\hline $\mathrm{P}_{2} \mathrm{xP}_{4}$ & $29.2 * *$ & $-94.3 * *$ & $50.1 * *$ & $-30.0 * *$ & $53.3 * *$ & $-19.9 * *$ & $-16.0 * *$ & $30.0 * *$ \\
\hline $\mathrm{P}_{2} \mathrm{XP}_{5}$ & $54.4^{* *}$ & $22.5^{* *}$ & $28.4^{* *}$ & $-56.6^{* *}$ & $3.7 * *$ & $-36.2^{* *}$ & $14.3 * *$ & $34.5^{* *}$ \\
\hline $\mathrm{P}_{3} \mathrm{xP}_{4}$ & $-7.7 * *$ & -1.2 & $-79.5 * *$ & $-70.3 * *$ & $-26.4 * *$ & $-68.5^{* *}$ & $-81.8 * *$ & $-42.0 * *$ \\
\hline $\mathrm{P}_{3} \mathrm{xP}_{5}$ & $44.4 * *$ & $35.6 * *$ & $-56.8 * *$ & $-17.3 * *$ & $-15.9 * *$ & $-17.3^{* *}$ & $7.0 * *$ & $11.1 * *$ \\
\hline $\mathrm{P}_{4} \mathrm{xP}_{5}$ & $22.1 * *$ & $14.3 * *$ & $13.7 * *$ & $-17.1 * *$ & $11.2 * *$ & $-28.9 * *$ & $-10.1 * *$ & $-6.5 * *$ \\
\hline
\end{tabular}

*** Significant at 0.05 and 0.01 probability levels, respectively.

\section{$\underline{\text { References }}$}

Abdalla, M.M.F., Shafik, M.M., Attia, Sabah M. and Ghannam, Hend A. (2017) Combining ability, heterosis and inbreeding effects in faba bean (Vicia faba L.). Journal of Experimental Agriculture International, 15(5), 1-13. Article no.JEAI.31964

Abdelmula, A.A., Link, W., Kittlitz, E. and von Stelling, D. (1999) Heterosis and inheritance of drought tolerance in faba bean Vicia faba L. Plant Breed. 118, 485-490.

Abdelmula, A.A., Link, W., Mohamed, A.A., Gasimand, S.M. and E.Khalifa, J. (2012) Genotypic variability in faba bean (Vicia faba L.) for Seed yield and protein content under drought stress during vegetative and reproductive stages. U.K.J. Agric. Sci. 20(1), 1-25.

Abd El-Zaher, I.N. (2016) Inheritance of seed yield and some yield components of faba bean using six populations. Assiut J. Agric. Sci. 47(4), 32-40.

Abid, G., Hessini, K., Aouida, M., Aroua, I., Baudoin, J., Muhovski, Y., Mergeai, G., Sassi, K., Machraoui, M., Souissi, F. and Jebara, M. (2017) Agro-physiological and biochemical responses of faba bean (Vicia faba L. var. 'minor') genotypes to water deficit stress. Biotechnol. Agron. Soc. Environ. 21(2), 146-159.

Ahmad, M.S.H. and Hager, M.A. (2010) Genetical analysis of some local and foreign faba bean cultivars. Plant Prod. Mansoura Univ. 1(12), 1679-1690.

Alghamdi, S.S. (2007) Genetic behavior of some selected faba bean genotypes, Proc. ${ }^{8}$ th Conf. African Crop Sci., El Minia, Egypt, pp. 709-714.

Alghamdi, S.S. (2009) Heterosis and combining ability in a diallel cross of eight faba bean (Vicia faba L.) genotypes. Asian J. Crop Sci. 1(2), 66-760.

Allen, R.G., Pereira, L.S., Raes, D. and Smith, M. (1998) Crop Evapotranspiration Guidelines for Computing Crop Water Requirements (Irrigation and drainage paper 56). FAO of the United Nations, Rome, Italy.

Ammar, M.H., Anwar, F., El-Harty, E.H., Migdadi, H.M., Abdel-Khalik, S.M., Al-Faifi, S.A., Farooq, M. and Alghamdi, S.S. (2014) Physiological and yield responses of faba bean (Vicia faba L.) to drought stress in managed and open field environments. J. Agro. Crop. Sci. ISSN 0931-2250.

Ammar, M.H., Khan, A.M., Migdadi, H.M., Abdelkhalek, S.M. and Alghamdi, S.S. (2017) Faba bean drought responsive gene identification and validation. Saudi Journal of Biological Sciences, 24, 80-89.

Ashrei, A.A.M., Rabi, E.M., Shafei, W.W.M., ELGarhy, A.M. and Abo-Mostafa, R.A. (2014) 
Performance and analysis of F1 and F2 diallel crossses among six parents of faba bean. Egypt. J.Plant Breed. 18(1),125-137.

Attia, S.M. and Salem, M.M. (2006) Analysis of yield and its components using diallel matings among five parents of faba bean. Egypt. J. Plant Breed. 10, $1-12$.

Bhatt, G.M. (1971) Heterosis performance and combining ability in a diallel cross among spring wheat (Triticum astivum L.). Australian J. Agric. Res. 22, 329-368.

Beyene, A.T., Derera, J., Sibiya, J. and Fikre, A. (2016) Gene action determining grain yield and chocolate spot (Botrytis fabae) resistance in faba bean. Euphytica, 702, 293-304.

Bishnoi, S.K., Yadav, I.S. and Panchta, R. (2012) Advances inheterosisand hybrid breeding in fababean (Vicia faba L.). Forage Res. 38(2), 24-27.

Bishnoi, S.K. (2016) Genetic diversity in relation to heterosis and combining ability in faba bean (Vicia faba L.). Ph. D. Thesis, College of Agriculture CCS Haryana Agricultural University. HISAR-125004 (Haryana).

Bishnoi, S.K., Hooda, J.S. and Sharma, P. (2018a) Analysis of gene effects for yield and yield component traits in faba bean (Vicia faba L.) genotypes. J. Anim. Plant. Sci. 28(1), 187-196.

Bishnoi, S.K., Hooda, J.S., Sharma, P. and Kumar, P. (2018b) Analysis of combining ability and inheritance of breeding parameters in yield component traits in faba bean (Vicia faba L.). Journal of Pharmacognosy and Phytochemistry, 7(2), 1085-1090.

Boghdady, M.S. (2009) Physiological and anatomical studies on mung bean plant under salinity conditions. Ph.D. Thesis. Faculty of Agriculture, Zagazig University, Egypt, 222p.

Bray, E.A. (1997) Plant responses to water deficit. Trends Plant Sci. 2, 48-54.

Chaves, M.M., Maroco, J.P. and Pereira, J.S. (2003) Understanding plant responses to drought- from genes to the whole plant. Funct. Plant Biol. 30, 239264.
Cortès, A.J., Monserrate, F.A., Ramírez-Villegas, J., Madriñán, S. and Blair, M.W. (2013) Drought tolerancein wild plant populations: The case of common beans (Phaseolus. vulgaris L.). PLoS ONE, 8, 1-10.

Darwish, D.S., Abdalla, M.M.F., El-Hady, M.M. and El-Emam, E.A.A. (2005) Investigations on faba beans, (Vicia faba L.) 19- Diallel and Triallel mating using five parents. Egypt. J. Plant Breed. 9, 197-208.

El-Bramawy, M.A.S. and Osman, M.A.M. (2012) Diallel crosses of genetic enhancement for seed yield components and resistance to leaf miner and aphid infestations of (Vicia faba L.). Int. J. Agr. Agr. R. 2(2), 8-21.

El-Hady, M.M., Olaa, S.M., El.-Galaly, A.M. and Salem, M.M. (2006) Heterosis and combining ability analysis of some faba bean genotypes. $J$. Agric. Res. Tanta Univ. 32, 134-148.

Fadeel, A.A. (1962) Location and properties of chloroplast and pigment determination in roots. Physiol. Plant, 15, 130-147.

Farag, H.I.A. and Afiah, S.A. (2012) Analysis of gene action in diallel crosses among some faba bean (Vicia faba L.) genotypes under Maryout conditions. Annals of Agricultural Science, 57(1), $37-46$.

Farooq, M, Basra, S.M.A., Wahid, A., Cheema, Z.A., Cheema, M.A. and Khaliq, A. (2008) Physiological role of exogenously applied glycinebetaine in improving drought tolerance of fine grain aromaticrice (Oryza. sativa L.). J. Agron. Crop Sci. 194, 325-333.

Farooq, M., Hussain, M., Wahid, A. and Siddique, K.H.M. (2012) Drought stress in plants: An overview. In: "Plant Responses to Drought Stress from Morphological to Molecular Features", Aroca, R. (Ed.), pp. 1-36. Springer-Verlag: Berlin, Germany.

Forouzan, B., Ahmad, A. and Hajar, A. (2013) Leaf anatomical characteristics in safflower genotypes as affected by drought stress. Acta Biologica Szegediensis, 57(1), 39-42.

Griffing, G.B. (1956) Concept of general and specific combining ability in relation to diallel crossing 
system. Australian Journal of Biological Sciences, 9, 463-493.

Haridy, A.G.H. and Amein, K.A. (2011) The inheritance of some agronomical traits, protein content and seed beetle (Collosobrucus maculatus) infestation in faba bean. Austral. J. Basic and Appl. Sci. 6, 1215-1222.

Hayman, B.I. (1958) The theory and analysis of diallel crosses II. Genetics, 42, 63-85.

Hazem, A.O.A., Naheif, E.M.M., Ahmed, A.G. and Mohamed, H.Z. (2013) Heterosis and nature of gene action for yield and its components in faba bean (Vicia faba L.). Journal of Plant Breeding and Crop Science, 5(3), 34-40.

Ibrahim, H.M. (2010) Heterosis, combining ability and components of genetic variance infaba bean (Vicia faba L.). JKAU Met. Environ. Arid Land Agric. Sci. 21(1), 35-50.

Ismail, A.I. (2016) Genetic behavior of earliness and yield characters of some faba bean (Vicia faba L.) genotypes. Ph. D. Thesis, Fac. of Agric., Zagazig University, Zagazig, Egypt.

Jaleel, C.A., Sankar, B., Murali, P.V., Gomathinayagam, M., Lakshmanan, G.M.A. and Panneerselvam, R. (2008) Water deficit stress effects on reactive oxygen metabolism in Catharanthus roseus; impacts on a jmalicine accumulation. Colloids Surf. $B, 62,105-111$.

Khan, H.R., Paull, J.G., Siddique, K.H.M. and Stoddard, F.L. (2010) Faba bean breeding for drought-affected environments: A physiological and agronomic perspective. Field Crops Research, 115, 279-286.

Khazaei, H. (2014) Leaf traits associated with drought adaptation in faba bean (Vicia faba L.). Ph. D. Thesis. Department of Agricultural Sciences Faculty of Agriculture and Forestry, University of Helsinki.

Link, W., Balko, C. and Stoddard, F.L. (2010) Winter hardiness in faba bean: Physiology and breeding. Field Crops Res. 115(3), 287-296.

Loss, S.P. and Siddique, K.H.M. (1997) Adaptation of faba bean (Vicia faba L.) to dryland Mediterraneantype environments I. Seed yield and yield components. Field Crop. Res. 53, 17-28.

Mather, K. and Jinks, J.L. (1982) "Biometrical Genetics", $3^{\text {rd }}$ ed. Chapman and Hall Ltd., London, ISBN-10: 0412228904.

Mohamed, B.A. (2015) Physiological response of German winter faba bean (Vicia faba L.) to drought. Journal of Crop Improvement, 29, 319-332.

Nassar, M.A. and El-Sahhar, K.F. (1998) "Botanical Preparations and Microscopy (Microtechnique)". Academic Bookshop, Dokki, Giza, Egypt, (In Arabic).

Omar, S.A. (2004) Breeding faba bean for environmental stress conditions, 2- Performance and phenotypic stability for yield and its components. Annals of Agric. Sci., Moshtohor, 42(1), 15-23.

Ping, L., Zhang, Y., Wu, X. and Liu, Y. (2018) Drought stress impact on leaf proteome variations of faba bean (Vicia faba L.) in the Qinghai-Tibet Plateau of China. 3 Biotech. 8, 110.

Petrov, P.I., Kocheva, K.V., Petrova, A.S. and Georgive, G.I (2012) Ion leakage and leaf anatomy of barley plant subjected to dehydration. Genetics and Plant Physiology, 2(1-2), 15-23.

Peyman, S. (2015) Genetic variation for seed yield and some of agro-morphological traits in faba bean (Vicia faba L.) genotypes. Acta Agriculturae Slovenica, 105(1), 73-83. DOI: 10.14720/aas.105.1.08.

Reddy, M.P. and Vora, A.B (1986) Changes in pigment composition, Hill reaction activity and saccharides metabolism in Bajra (Pennisetum typhoides $\mathrm{S} \& \mathrm{H}$ ) leaves under $\mathrm{NaCl}$ salinity. Photosynthetica, 20, 50-55.

Selim, A.F.H. and El-Nady, M.F. (2011) Physioanatomical responses of drought stressed tomato plants to magnetic field. Acta Astronautica, 69, 387-396.

Siddiqui, M.H., Al-Khaishany, M.Y., Al-Qutami, M.A., Al-Whaibi, M.H., Grover, A., Ali, H.M., AlWahibi, M.S. and Bukhari, N.A. (2015) Response of different genotypes of faba bean plant to drought stress. Int. J. Mol. Sci. 16, 10214-10227. doi:10.3390/ijms160510214.

Steel, R.G.D. and Torrie, T. (1980) "Principles and 
Procedures of Statistics". A biometrical Approach. Mc Graw Hill Book Co., Inc., New York.

Ukai, Y. (2002) Genetic analysis of quantitative traits controlled by several major genes by means of diallel crosses. IgakuShuppan. Tokyo. (In Japanese). http://peach.ab.a.u-tokyo.ac.jp/ ukai/.
Zeinab, E. and Helal, A.G. (2014) Diallel analysis and separation of genetic variance components in eight faba bean genotypes. Annals of Agricultural Science, 59(1), 147-154

(Received 8/ 8/2018; accepted 4/12/2018)

\section{تقديرات القدرة الائتلافية وكفاءة التوريث وقوة الهجين فى الفول البلدى تحت الإجهاد المائى}

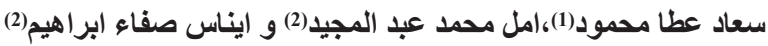

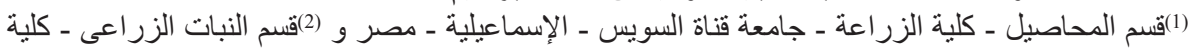
الزر اعة - جامعة قناة السويس ـ الإسماعيلية ـ مصر.

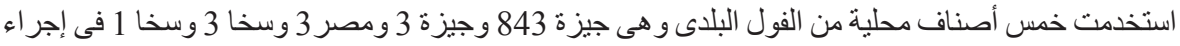

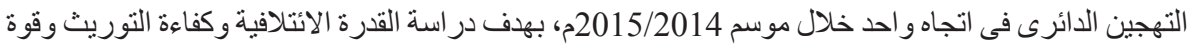

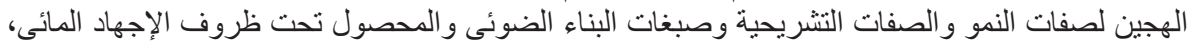

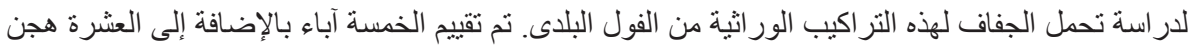

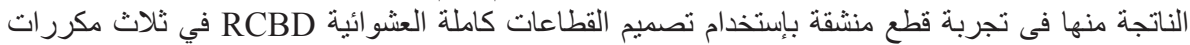

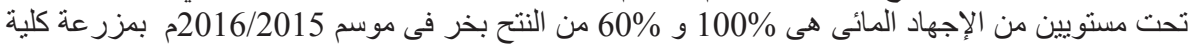

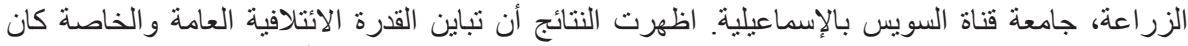

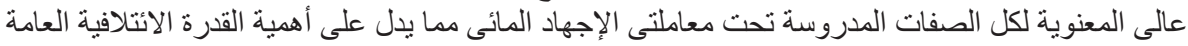

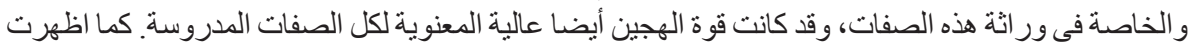

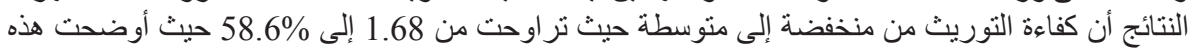

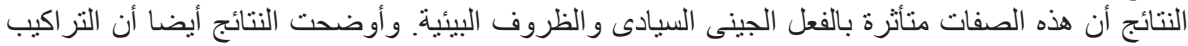

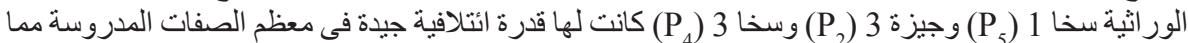

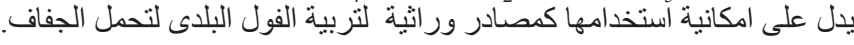

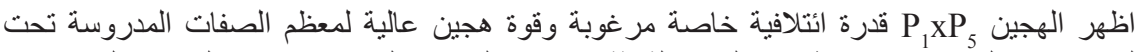

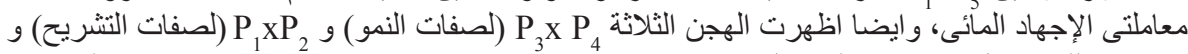

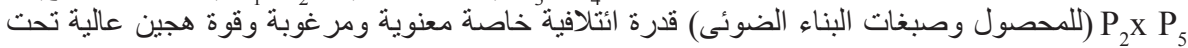

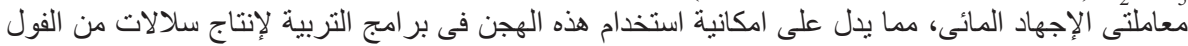

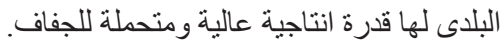

